\begin{tabular}{|c|c|}
\hline Title & High-Dimensional Graphical Networks of Self-A voiding W alks \\
\hline Author(s) & Holmes, Mark; Járai, A ntal A .; Sakai, A kira; Slade, Gordon \\
\hline Citation & $\begin{array}{l}\text { Canadian Journal of Mathematics, } 56(1), 77-114 \\
\text { https://doi.org/10.4153/CJM-2004-005-1 }\end{array}$ \\
\hline Issue Date & $2004-02-01$ \\
\hline Doc URL & http:/hdl. handle.net/2115/58575 \\
\hline Rights & $\begin{array}{l}\text { A rchived with the permission of the Canadian Mathematical Society, this article was originally published in the } \\
\text { Canadian Journal of Mathematics by Mark Holmes, A kira Sakai, A ntal A. Jarai and Gordon Slade, High-Dimensional } \\
\text { Graphical Networks of Self-A voiding W alks, Vol.56 no.1 (2004), pg 77-114, DOI:10.4153/ JM-2004-005-1. }\end{array}$ \\
\hline Type & article \\
\hline File Information & CanadJMath_56_p77-.pdf \\
\hline
\end{tabular}

Instructions for use 


\title{
High-Dimensional Graphical Networks of Self-Avoiding Walks
}

\author{
Mark Holmes, Antal A. Járai, Akira Sakai and Gordon Slade
}

Abstract. We use the lace expansion to analyse networks of mutually-avoiding self-avoiding walks, having the topology of a graph. The networks are defined in terms of spread-out self-avoiding walks that are permitted to take large steps. We study the asymptotic behaviour of networks in the limit of widely separated network branch points, and prove Gaussian behaviour for sufficiently spread-out networks on $\mathbb{Z}^{d}$ in dimensions $d>4$.

\section{Introduction and Results}

\subsection{Introduction}

A single self-avoiding walk is often used as a model of a linear polymer in a good solution. Networks of mutually-avoiding self-avoiding walks can be used to model networks of polymers containing monomers capable of making more than two chemical bonds, leading to branching. The rich critical behaviour of polymer networks has been studied in the physics literature [2], but is mainly open mathematically.

Recently [5], the lace expansion was used to prove Gaussian behaviour for sufficiently spread-out networks of mutually-avoiding self-avoiding walks on $\mathbb{Z}^{d}$, for networks with the topology of a tree in dimensions $d>4$. The results of [5] are in the limit as the length of the self-avoiding walks comprising the network grow to infinity. In this paper, we consider networks with the topology of a general graph. We study the asymptotic behaviour of networks consisting of self-avoiding walks of arbitrary length, weighted at criticality, in the limit in which the network's branch points are fixed at lattice sites that are widely separated. The graph giving the topology of the network may contain cycles or multiple edges between vertices. Edges joining a vertex to itself (self-lines) are not permitted, as these are not relevant in the limit we study.

We prove Gaussian behaviour for sufficiently spread-out graphical networks on $\mathbb{Z}^{d}$ in dimensions $d>4$. The proof is based on the lace expansion on a tree [5], but major modifications are required to extend the expansion from a tree to a general graph. The proof is also based on the result of [4] that the critical two-point function for sufficiently spread-out self-avoiding walks on $\mathbb{Z}^{d}$, with $d>4$, decays like a multiple of $|x|^{2-d}$ as $|x| \rightarrow \infty$.

Received by the editors April 25, 2002; revised November 6, 2002.

AMS subject classification: Primary: 82B41; secondary: 60K35.

(C) Canadian Mathematical Society 2004. 


\subsection{Networks}

The term graph will be reserved for graphs that arise in the lace expansion. We therefore refer to the underlying graph that determines our network as a shape. A shape $\nu=(S, E)$ consists of a finite set $S$ of vertices and a finite set $E$ of directed pairs $e=\left(e_{1}, e_{2}\right)$ of vertices (edges). We allow repeated elements in $E$ (multiple lines) but do not allow $e \in E$ with $e_{2}=e_{1}$ (no self-lines). We do not assume that $\nu$ is connected; our methods and results apply whether $\nu$ is connected or not. Given a shape $\nu$, we call one of its vertices the root and label it by 0 . The remaining vertices are labelled in a fixed but arbitrary manner as $1, \ldots,|S|-1$. We also label the edges in a fixed but arbitrary manner, as $1, \ldots,|E|$.

For $\vec{n}=\left(n_{1}, \ldots, n_{|E|}\right) \in \mathbb{N}|E|$, we let $\mathcal{N}=\mathcal{N}(\nu, \vec{n})$ denote the subdivision of $\nu$ obtained by inserting $n_{e}-1$ vertices on each $e \in E$. We refer to the inserted vertices as path points, and to the remaining vertices of $\mathcal{N}$, which are identical to the original vertices in $S$, as branch points. We denote the degree of a branch point $i$ by $\Delta_{i}$. We write $\mathcal{S}$ for the set of vertices of $\mathcal{N}$ and $\mathcal{E}$ for the set of edges of $\mathcal{N}$, so that $\mathcal{N}=(\mathcal{S}, \mathcal{E})$.

A network is a mapping $\omega: \mathcal{S} \rightarrow \mathbb{Z}^{d}$ (although we occasionally refer also to $\mathcal{N}$ as a network). The relation between the shape $\nu$, its subdivision $\mathcal{N}$, and the embedding into $\mathbb{Z}^{d}$ by $\omega$ is indicated in Figure 1. We require that $\omega$ maps the root 0 to the origin in $\mathbb{Z}^{d}$. At this point, we do not assume that $\omega$ is one-to-one. To each embedding we associate a weight. This weight is defined in terms of a parameter $z>0$, and a function $D: \mathbb{Z}^{d} \rightarrow[0,1]$ of the form given in Definition 1.1 below, by

$$
W_{z}(\omega)=\prod_{e \in \mathcal{E}} z D\left(\omega\left(e_{2}\right)-\omega\left(e_{1}\right)\right)
$$

In the degenerate case of a network consisting of a single vertex and no edges, we interpret the empty product in (1.1) as 1. Definition 1.1 involves a positive parameter $L$, which serves to spread out the embeddings, and which we take to be large, providing a small parameter $L^{-1}$.

Definition 1.1 Let $h$ be a non-negative bounded function on $\mathbb{R}^{d}$ which is piecewise continuous, invariant under the lattice symmetries of reflection in coordinate hyperplanes and rotation by $90^{\circ}$, supported in $[-1,1]^{d}$, and normalised so that $\int_{[-1,1]^{d}} h(x) d^{d} x=1$. Then for large $L$ we define

$$
D(x)=\frac{h(x / L)}{\sum_{x \in \mathbb{Z}^{d}} h(x / L)} .
$$

Since $\sum_{x \in \mathbb{Z}^{d}} h(x / L) \sim L^{d}$ (using a Riemann sum approximation to $\int_{[-1,1]^{d}} h(x) d^{d} x$ ), the assumption that $L$ is large ensures that the denominator of (1.2) is nonzero. We also define $\sigma^{2}=\sum_{x}|x|^{2} D(x)$.

The sum $\sum_{x}|x|^{p} D(x)$ can be regarded as a Riemann sum, and is asymptotic to a multiple of $L^{p}$ for $p>0$. In particular, $\sigma$ and $L$ are comparable. A basic example obeying the conditions of Definition 1.1 is given by the function $h(x)=2^{-d}$ for 
$x \in[-1,1]^{d}, h(x)=0$ otherwise, for which $D(x)=(2 L+1)^{-d}$ for $x \in[-L, L]^{d} \cap$ $\mathbb{Z}^{d}, D(x)=0$ otherwise. For this example, $W_{z}(\omega)$ is either $\left[z(2 L+1)^{-d}\right]^{|\mathcal{E}|}$ or 0 , depending on whether or not $\left\|\omega\left(e_{2}\right)-\omega\left(e_{1}\right)\right\|_{\infty} \leq L$ for every edge $e=\left(e_{1}, e_{2}\right) \in \mathcal{E}$.

We refer to an undirected pair $\{s, t\}$ of distinct vertices in $\mathcal{S}$ as a bond, and write simply st for $\{s, t\}$. Let $\mathcal{B}_{\mathcal{N}}$ denote the set of bonds of $\mathcal{N}$. Given $b=s t \in \mathcal{B}_{\mathcal{N}}$ and an embedding $\omega$, let

$$
U_{b}(\omega)=U_{s t}(\omega)=-\mathbb{1}_{[\omega(s)=\omega(t)]},
$$

where $\mathbb{1}_{[\cdot]}$ is the indicator function. The product $\prod_{b \in \mathcal{B}_{N}}\left[1+U_{b}(\omega)\right]$ is nonzero if and only if the embedding $\omega$ is one-to-one. In other words, this product is nonzero precisely when the network of embedded walks specified by $\omega$ is a self-avoiding network.

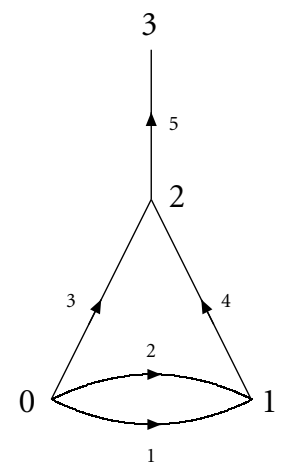

$\nu$

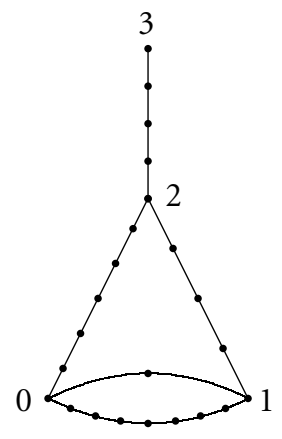

$\mathcal{N}(\nu, \vec{n})$

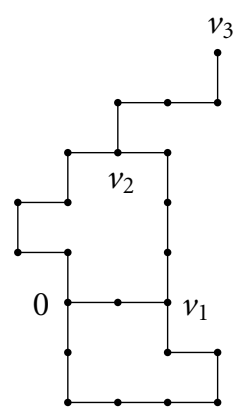

Figure 1: A shape $\nu$ and subdivision $\mathcal{N}(\nu, \vec{n})$ with $\vec{n}=(8,2,6,4,4)$, and a nearest-neighbour self-avoiding network in $\Omega_{\mathcal{N}}(\vec{x})$ with $\vec{x}=((2,0),(2,0),(1,3),(-1,3),(2,2))$; see (1.4)(1.5).

Given $\mathcal{N}$ and an embedding $\omega$, we denote the location of the embedded branch points by

$$
v_{i}=\omega(i) \quad(i \in S) .
$$

Given $\vec{x}=\left(x_{1}, \ldots, x_{|E|}\right) \in \mathbb{Z}^{d|E|}$, we denote by $\Omega_{\mathcal{N}}(\vec{x})$ the set of embeddings such that

$$
v_{e_{2}}-v_{e_{1}}=x_{e} \quad\left(e=\left(e_{1}, e_{2}\right) \in E\right)
$$

Note that $\vec{x}$ uniquely determines $\vec{v}=\left(v_{0}, \ldots, v_{|S|-1}\right)$. Our object of study is

$$
G_{\nu}(\vec{x})=\sum_{\vec{n} \in \mathbb{N}^{|E|}} \sum_{\omega \in \Omega_{\mathcal{N}}(\vec{x})} W_{z_{\mathcal{c}}}(\omega) \prod_{b \in \mathcal{B}_{\mathcal{N}}}\left[1+U_{b}(\omega)\right],
$$


where $z_{c}$ is chosen to be the critical value of $z$. The critical value is defined as follows. Let $\nu_{1}$ denote the shape consisting of a single edge joining two vertices. The selfavoiding walk two-point function is defined by

$$
G(x)=\delta_{0, x}+G_{\nu_{1}}(x)
$$

with $G_{\nu_{1}}(0)=0$. With $z_{c}$ replaced by $z$ in the definition of $G_{\nu_{1}}(x)$ in (1.6)-(1.7), it is well-known that there is a critical value $z_{c}$ such that $\sum_{x \in \mathbb{Z}^{d}} G(x)$ converges for $z<z_{c}$ and diverges for $z \geq z_{c}$ (see, e.g., [4]). We use this value $z_{c}$ in (1.6).

The asymptotic behaviour of $G(x)$ was determined in [4], for $d>4$ and $L$ sufficiently large. (For a related result for nearest-neighbour self-avoiding walks in very high dimensions, see [3].) To state the result of [4], we define

$$
\epsilon_{2}=2(d-4) \wedge 2
$$

For $d \geq 5, \epsilon_{2}$ is simply equal to 2 . However, it is instructive to keep the $d$-dependence explicit in (1.8) to reveal the critical nature of $d=4$. Theorem 1.2 presumably holds with $\alpha=0$, but it has been proved only for $\alpha>0$. We think of $\alpha$ as small.

Theorem 1.2([4]) Let $d>4$ and fix any $\alpha>0$. There is a finite constant $A=$ $A(d, L)$, and an $L_{0}=L_{0}(d, \alpha)$, such that for $L \geq L_{0}$,

$$
G(x)=\frac{A}{\sigma^{2}(|x| \vee 1)^{d-2}}\left[1+O\left(\frac{1}{(|x| \vee 1)^{\epsilon_{2}-\alpha}}\right)\right] .
$$

The amplitude A obeys $A=\frac{1}{2} d \Gamma\left(\frac{d}{2}-1\right) \pi^{-d / 2}+O\left(L^{-2+\alpha}\right)$. In addition,

$$
G(x) \leq O\left(\frac{1}{L^{2-\alpha}(|x| \vee 1)^{d-2}}\right) \quad(x \neq 0) .
$$

The constant in the error term in (1.9) depends on $\alpha$ and $L$, while the constant in (1.10) depends on $\alpha$ but not on $L$.

In this paper, we extend Theorem 1.2 to general networks.

\subsection{Main Result}

Our main result is the following theorem.

Theorem 1.3 Let $d>4$. Fix $\nu=(S, E)$ and $\epsilon_{1}<(d-4) \wedge 1$. There exist $L_{0}=$ $L_{0}(d, \nu)$ and constants $V_{\Delta}=V_{\Delta}(d, L)$ such that for $L \geq L_{0}$,

$$
G_{\nu}(\vec{x})=\left[\prod_{i \in S} V_{\Delta_{i}}\right] \prod_{e \in E} \frac{A}{\sigma^{2}\left(\left|x_{e}\right| \vee 1\right)^{d-2}}\left[1+\sum_{i, j \in S: i \neq j} O\left(\frac{1}{\left(\left|v_{i}-v_{j}\right| \vee 1\right)^{\epsilon_{1}}}\right)\right],
$$

where $\vec{v}$ and $\vec{x}$ are related by (1.5). Constants in the error term depend on $L, d, \nu$ and $\epsilon_{1}$. 
The constants $V_{\Delta}$ appearing in the theorem will be referred to as vertex factors, and are identical to the vertex factors in [5]. By definition, $V_{1}=1$. It is proved in [5] that $V_{\Delta}=1+O\left(L^{-d}\right)$ for $\Delta \geq 2$. Theorem 1.3 states that the leading asymptotic behaviour of the network is that of a network of independent self-avoiding walks, apart from the vertex factors. Each vertex factor takes into account the local effect of the mutual avoidance of the walks that meet at that vertex. The mutual avoidance diminishes the number of allowed configurations, and this is reflected by the fact that there is a positive constant $c_{\Delta}$ such that

$$
V_{\Delta} \leq 1-c_{\Delta} L^{-d} \quad(\Delta \geq 2) .
$$

Although not explicitly stated in [5], (1.12) follows easily from the results of [5].

Theorem 1.3 gives the leading behaviour of $G_{\nu}(\vec{x})$ in the limit where the embedded network branch points are widely separated, i.e., in the limit $\min _{i, j \in S}\left|v_{i}-v_{j}\right| \rightarrow \infty$. Note that each $x_{e}$ is equal to some $v_{i}-v_{j}$, so this limit requires $\left|x_{e}\right| \rightarrow \infty$ for all $e$, in particular. On the other hand, one cannot hope to exclude terms with nonadjacent $i, j \in S$ from the error term. This is because if $\left|v_{i}-v_{j}\right|$ is small, the effect of the interaction between the walks incident on $v_{i}$ with those incident on $v_{j}$ does not decouple into a product of $V_{\Delta_{i}}$ and $V_{\Delta_{i}}$.

Our proof of the theorem is restricted to large $L$, although we expect the result to remain true for all $L \geq 1$ (subject to a degree restriction on the network to allow for a one-to-one embedding). We have made no attempt to optimise the error estimate in (1.11), neither with respect to the power of $\left|v_{i}-v_{j}\right|$ nor the $L$-dependence. Theorem 1.3 is a new result also for networks with the shape of a tree, as the asymptotics in (1.11) involve a different limit than the one studied in [5].

The remainder of the paper is devoted to the proof of Theorem 1.3. We begin the proof in Section 2, where the graphical expansion leading to the lace expansion is described and the proof of Theorem 1.3 is reduced to several propositions.

\section{The Expansion and Proof of Theorem 1.3}

In this section, we perform an expansion of the factor $\prod_{b \in \mathcal{B}_{\mathcal{N}}}\left[1+U_{b}\right]$ in (1.6). Due to the possibility of cycles in the network, new difficulties arise that have not been encountered in previous work using the lace expansion. These difficulties are described and overcome in Section 2.1. The expansion is then used in Section 2.2 to rewrite $G_{\nu}(\vec{x})$ as a sum of a main term plus error terms. In Section 2.3, we state several propositions and use these propositions to prove Theorem 1.3.

\subsection{Classification of Bonds}

We refer to a set of bonds as a graph. For a network with the shape of a tree, each bond $s t$ determines an interval, namely the unique path in the tree joining $s$ and $t$. In the lace expansion, these intervals are used to define a notion of connectivity for graphs. However, if a shape contains a cycle, then a bond st with both $s$ and $t$ on the cycle does not determine a unique interval joining $s$ and $t$. This can be seen, for example, in the "bubble" shape of Figure 2. There are some bonds to which it will turn out to 
be unnecessary to associate an interval. But for many bonds we will need to associate an interval, and the choice of interval will be made according to the embedding $\omega$ of the network.

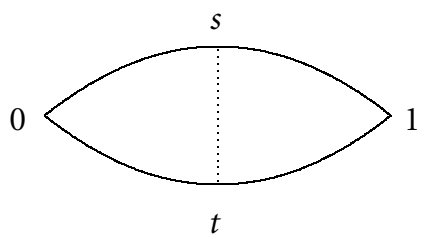

Figure 2: The solid lines represent the bubble shape $\nu$ consisting of vertices 0,1 joined by a pair of edges $(0,1),(0,1)$. The dotted line represents a bond $s t$ on a subdivision $\mathcal{N}$ of $\nu$. The bond st does not determine a unique interval on $\mathcal{N}$.

We describe this together with a classification of bonds that depends on the embedding $\omega$. Fix $\nu=(S, E), \vec{x}=\left(x_{1}, \ldots, x_{|E|}\right) \in \mathbb{Z}^{d|E|}$, and $\omega \in \Omega_{\mathcal{N}}(\vec{x})$. We denote by $T_{e}^{\circ}$ the set of path points of $\mathcal{N}$ that were inserted on the edge $e=\left(e_{1}, e_{2}\right)$ of $\nu$, and define $T_{e}=T_{e}^{\circ} \cup\left\{e_{1}, e_{2}\right\}$. For $w, x \in \mathbb{Z}^{d}$, let

$$
B_{w}(x)=\left\{y \in \mathbb{Z}^{d}:|y-w| \leq \frac{1}{3}|x|\right\}, \quad B(x)=B_{0}(x) .
$$

A vertex $u$ of $\mathcal{N}$ is said to be near a branch point $i$ if there is an edge $e$ incident on $i$ such that $u \in T_{e}^{\circ} \cup\{i\}$ and $\omega(u) \in B_{v_{i}}\left(x_{e}\right)$. We classify bonds as follows:

1. For $e \in E$, we denote by $\mathcal{H}_{\mathcal{N}, e}$ the set of bonds st with $s \in T_{e}, t \in T_{e}^{\circ}$. Note that $\mathcal{H}_{\mathcal{N}, e}$ is independent of $\omega$. Let $\mathcal{H}_{\mathcal{N}}=\dot{\bigcup}_{e \in E} \mathcal{H}_{\mathcal{N}, e}$. To $s t \in \mathcal{H}_{\mathcal{N}, e}$, we associate the interval consisting of the vertices in the path from $s$ to $t$ in $T_{e}$, excluding $s$ and $t$.

2. For $i \in S$, we denote by $\mathcal{V}_{\mathcal{N}, i}=\mathcal{V}_{\mathcal{N}, i}(\omega)$ the set of bonds $s t$ such that $s$ and $t$ are both near $i$, with $s \in T_{e}^{\circ}, t \in T_{e^{\prime}}^{\circ}$ for two different edges $e, e^{\prime}$ incident on $i$. Let $\mathcal{V}_{\mathcal{N}}=\dot{U}_{i \in S} \mathcal{V}_{\mathcal{N}, i}$. To $s t \in \mathcal{V}_{\mathcal{N}, i}$, we associate the interval consisting of the vertices in the path from $s$ to $t$ (excluding $s$ and $t$ ) which includes no branch point except $i$.

3. Let $\mathcal{R}_{\mathcal{N}}=\mathcal{R}_{\mathcal{N}}(\omega)=\mathcal{B}_{\mathcal{N}} \backslash\left(\mathcal{H}_{\mathcal{N}} \cup \dot{\mathcal{V}} \mathcal{V}_{\mathcal{N}}\right)$.

Note that a bond in $\mathcal{H}_{\mathcal{N}} \cup \mathcal{V}_{\mathcal{N}}$ either has both endpoints on the same branch $T_{e}$, or on adjacent branches. The classification is depicted in Figure 3.

Given $\omega$ and $\vec{x}$, the above classification partitions $\mathcal{B}_{\mathcal{N}}$ into the disjoint union

$$
\mathcal{B}_{\mathcal{N}}=\mathcal{V}_{\mathcal{N}} \cup \dot{\mathcal{H}_{\mathcal{N}}} \cup \dot{\mathcal{R}_{\mathcal{N}}}
$$

Therefore,

$$
\prod_{b \in \mathcal{B}_{\mathcal{N}}}\left[1+U_{b}\right]=\prod_{b \in \mathcal{V}_{\mathcal{N}} \cup \mathcal{H}_{\mathcal{N}}}\left[1+U_{b}\right]-\left[\prod_{b \in \mathcal{V}_{\mathcal{N}} \cup \mathcal{H}_{\mathcal{N}}}\left[1+U_{b}\right]\right]\left[1-\prod_{b \in \mathcal{R}_{\mathcal{N}}}\left[1+U_{b}\right]\right]
$$




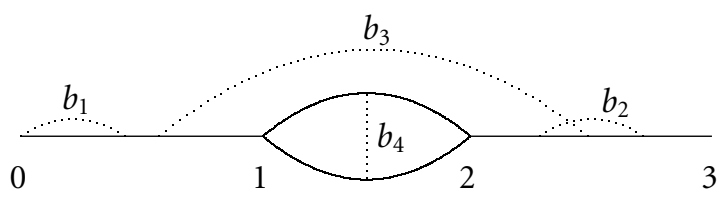

Figure 3: The solid lines represent a shape with branch points $0,1,2,3$. The dotted lines represent bonds on a subdivision $\mathcal{N}$ of the shape. The bonds depicted are classified as: $b_{1}, b_{2} \in \mathcal{H}_{\mathcal{N}}$; $b_{3} \in \mathcal{R}_{\mathcal{N}} ; b_{4} \in \mathcal{V}_{\mathcal{N}}$ if its endpoints are both near 1 or both near 2 , otherwise $b_{4} \in \mathcal{R}_{\mathcal{N}}$.

We define $\psi_{\nu}(\vec{x})$ by replacing $\prod_{b \in \mathcal{B}_{\mathcal{N}}}\left[1+U_{b}\right]$ in (1.6) with the second term of the right side of (2.3), i.e.,

$$
\psi_{\nu}(\vec{x})=-\sum_{\vec{n} \in \mathbb{N}^{|E|}} \sum_{\omega \in \Omega_{\mathcal{N}}(\vec{x})} W_{z_{c}}(\omega)\left[\prod_{b \in \mathcal{V}_{\mathcal{N}} \cup \mathcal{H}_{\mathcal{N}}}\left[1+U_{b}(\omega)\right]\right]\left[1-\prod_{b \in \mathcal{R}_{\mathcal{N}}}\left[1+U_{b}(\omega)\right]\right] .
$$

This will turn out to be an error term.

To gain some insight into the classification of bonds, consider $\psi_{\nu}(\vec{x})$ when $\nu$ has the bubble shape of Figure 2 and $\vec{x}=(x, x)$, so that one branch point is embedded at the origin of $\mathbb{Z}^{d}$ and the other is embedded at $x$. The final factor of (2.4) is nonzero only if there is a bond $b=\{s, t\} \in \mathcal{R}_{\mathcal{N}}$ such that $\omega(s)=\omega(t)$. This implies an intersection as indicated in Figure 4. When the intersection takes place outside the balls $B(x)$ and $B_{x}(x)$, as required by $\mathcal{R}_{\mathcal{N}}$, decay of the form $|x|^{-(3 d-8)}$ can be proved (see Proposition 2.4 below). This is smaller than the leading behaviour $|x|^{-(2 d-4)}$ given in Theorem 1.3, by a factor $|x|^{-(d-4)}$. On the other hand, when an intersection takes place close to 0 or $x$, we have a contribution to the vertex factor at that vertex and the same behaviour as the leading term.

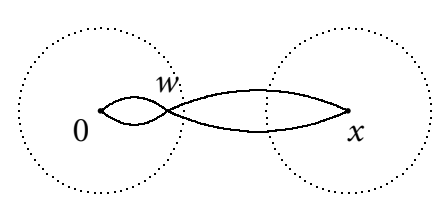

$w \in B(x)$

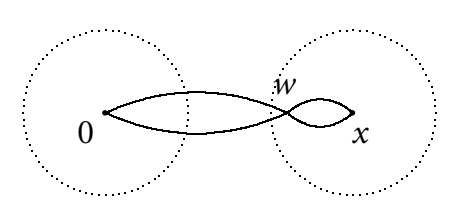

$w \in B_{x}(x)$

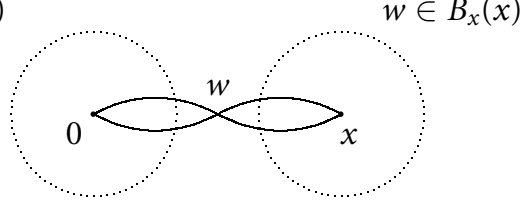

$w \notin B(x) \cup B_{x}(x)$

Figure 4: The bottom configuration contributes to an error term, whereas the other two contribute to the leading term. 


\subsection{Expansion and Extraction of the Main Term}

In this section, we extract the main contribution to $G_{\nu}(\vec{x})$ and obtain a representation for it. This requires the notion of a connected graph, which we will define for graphs consisting of bonds in $\mathcal{V}_{\mathcal{N}} \cup \mathcal{H}_{\mathcal{N}}$.

Definition 2.1 Let $\omega \in \Omega_{\mathcal{N}}(\vec{x})$ be fixed.

1. Let $\mathcal{G}_{\mathcal{N}}=\mathcal{G}_{\mathcal{N}}(\omega)$ denote the set of graphs on $\mathcal{N}$ consisting of bonds in $\mathcal{V}_{\mathcal{N}} \cup \mathcal{H}_{\mathcal{N}}$.

2. A bond $b \in \mathcal{V}_{\mathcal{N}} \cup \mathcal{H}_{\mathcal{N}}$ is said to cover all vertices in the interval associated to $b$ as in Section 2.1. In addition, if a vertex of $b$ is identical to a leaf of $\nu$, we say that $b$ covers that leaf. Given a graph $\Gamma \in \mathcal{G}_{\mathcal{N}}$, a path point $u$ is said to be fully covered by $\Gamma$ if there is a bond $b \in \Gamma$ covering $u$. A branch point $i$ is said to be fully covered by $\Gamma$ if there are bonds $s_{1} t_{1}, \ldots, s_{k} t_{k}$ in $\Gamma$ such that each one covers $i$ and such that each $T_{e}$ incident on $i$ has at least one vertex among $s_{1}, t_{1}, \ldots, s_{k}, t_{k}$. This requires $k \geq\left\lceil\frac{\Delta_{i}}{2}\right\rceil$. We say that $\Gamma$ is a connected graph on $\mathcal{N}$ if every vertex of $\mathcal{N}$ is fully covered by $\Gamma$.

3. Let $\mathcal{A}$ be a connected subnetwork of $\mathcal{N}$. Given $\Gamma \in \mathcal{G}_{\mathcal{N}}$, we denote by $\left.\Gamma\right|_{\mathcal{A}}$ the restriction of $\Gamma$ to $\mathcal{A}$, i.e., $\left.\Gamma\right|_{\mathcal{A}}$ consists of bonds $b \in \Gamma$ such that the endvertices of $b$ and all vertices covered by $b$ are in the set of vertices of $\mathcal{A}$.

Let $S_{0} \subset S$ denote the set of branch points that are not leaves. If $S_{0}=\varnothing$, then $\nu$ is a single edge or a union of disjoint edges, and Theorem 1.3 is implied by Theorem 1.2. We therefore restrict attention to shapes with $S_{0}$ nonempty.

Expanding the first term of (2.3) gives

$$
\prod_{b \in \mathcal{V}_{\mathcal{N}} \cup \mathcal{H}_{\mathcal{N}}}\left[1+U_{b}\right]=\sum_{\Gamma \in \mathcal{G}_{\mathcal{N}}} \prod_{b \in \Gamma} U_{b}
$$

where the empty product is 1 . Given a graph $\Gamma \in \mathcal{G}_{\mathcal{N}}$ and a branch point $i \in S_{0}$, we denote by $\mathcal{A}_{i}=\mathcal{A}_{i}(\Gamma)$ the largest connected subnetwork of $\mathcal{N}$ that includes $i$ (not as a leaf of $\mathcal{A}_{i}$ ), such that $\left.\Gamma\right|_{\mathcal{A}_{i}}$ is a connected graph on $\mathcal{A}_{i}$. If $\Gamma$ does not contain a bond that covers $i$, then we set $\mathcal{A}_{i}=\{i\}$. We define $\mathcal{G}_{\mathcal{N}}^{\text {main }}=\mathcal{G}_{\mathcal{N}}^{\text {main }}(\omega)$ to be the set of graphs in $\mathcal{G}_{\mathcal{N}}$ such that no $\mathcal{A}_{i}$ contains more than one element of $S_{0}$, except possibly as a leaf. Let $\mathcal{G}_{\mathcal{N}}^{\text {err }}=\mathcal{G}_{\mathcal{N}} \backslash \mathcal{G}_{\mathcal{N}}^{\text {main }}$. See Figure 5. Then

$$
\prod_{b \in \mathcal{V}_{\mathcal{N}} \cup \mathcal{H}_{\mathcal{N}}}\left[1+U_{b}\right]=\sum_{\Gamma \in \mathcal{G}_{\mathcal{N}}^{\text {main }}} \prod_{b \in \Gamma} U_{b}+\sum_{\Gamma \in \mathcal{G}_{\mathcal{N}}^{\text {err }}} \prod_{b \in \Gamma} U_{b}
$$

We define $M_{\nu}(\vec{x})$ and $\varphi_{\nu}(\vec{x})$ by

$$
\begin{aligned}
& M_{\nu}(\vec{x})=\sum_{\vec{n} \in \mathbb{N}|E|} \sum_{\omega \in \Omega_{\mathcal{N}}(\vec{x})} W_{z_{\mathcal{c}}}(\omega) \sum_{\Gamma \in \mathcal{G}_{\mathcal{N}}^{\text {main }}} \prod_{b \in \Gamma} U_{b}(\omega), \\
& \varphi_{\nu}(\vec{x})=\sum_{\vec{n} \in \mathbb{N}^{|E|}} \sum_{\omega \in \Omega_{\mathcal{N}}(\vec{x})} W_{z_{\mathcal{c}}}(\omega) \sum_{\Gamma \in \mathcal{G}_{\mathcal{N}}^{\text {err }}} \prod_{b \in \Gamma} U_{b}(\omega) .
\end{aligned}
$$



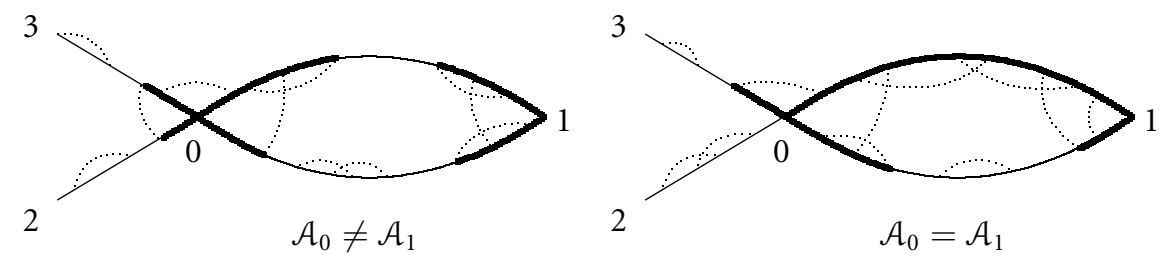

Figure 5: Contributions to $\mathcal{G}_{\mathcal{N}}^{\text {main }}$ and $\mathcal{G}_{\mathcal{N}}^{\text {err }}$, using the convention that a bond is drawn to cover the interval on its concave side. The subnetworks $\mathcal{A}_{0}$ and $\mathcal{A}_{1}$ are indicated in bold.

Therefore, recalling (2.3)-(2.4) and (1.6),

$$
G_{\nu}(\vec{x})=M_{\nu}(\vec{x})+\varphi_{\nu}(\vec{x})+\psi_{\nu}(\vec{x}) .
$$

The main term in (2.9) is $M_{\nu}(\vec{x})$, and we now analyse this term further.

Let $\tau_{\Delta}$ denote the star shape consisting of a single vertex of degree $\Delta$ (the root) joined to $\Delta$ leaves by $\Delta$ edges. In particular, $\tau_{0}$ consists of a single vertex and no edges. Given $\Gamma \in \mathcal{G}_{\mathcal{N}}^{\text {main }}$, to each branch point $i \in S_{0}$ there corresponds a star-shaped subnetwork $\mathcal{A}_{i}$, as described above. We denote the leaves of $\mathcal{A}_{i}$ by $a_{1}^{(i)}, \ldots, a_{\Delta_{i}}^{(i)}$, whose ordering obeys the original ordering of edges in $E$. Each $a_{k}^{(i)}$ may coincide with $i$. However, $\mathcal{A}_{i}$ cannot have degree 1 at $i$, by definition. Thus $\mathcal{A}_{i}$ is star shaped of degree $\Delta_{i}^{\prime}$ for some $\Delta_{i}^{\prime} \in\left\{0,2,3, \ldots, \Delta_{i}\right\}$. For $e=\left(e_{1}, e_{2}\right) \in E$, let $\mathcal{J}_{e}=\mathcal{J}_{e}(\Gamma)$ denote the interval on $T_{e}$ between $\mathcal{A}_{e_{1}}$ and $\mathcal{A}_{e_{2}}$, including the leaves of $\mathcal{A}_{e_{1}}$ and $\mathcal{A}_{e_{2}}$ on $T_{e}$. See Figure 6. Then

$$
\sum_{\Gamma \in \mathcal{G}_{\mathcal{N}}^{\text {main }}} \prod_{b \in \Gamma} U_{b}=\sum_{\Gamma \in \mathcal{G}_{\mathcal{N}}^{\text {main }}}\left[\prod_{i \in S_{0}}\left[\prod_{\left.b \in \Gamma\right|_{\mathcal{A}_{i}}} U_{b}\right]\right] \prod_{e \in E}\left[\prod_{\left.b \in \Gamma\right|_{J_{e}}} U_{b}\right]
$$

In (2.10), we isolate the connected component of $\Gamma$ around each vertex in $S_{0}$. This is different than the approach of [5], where a connected component of $\Gamma$ is isolated at only one branch point.
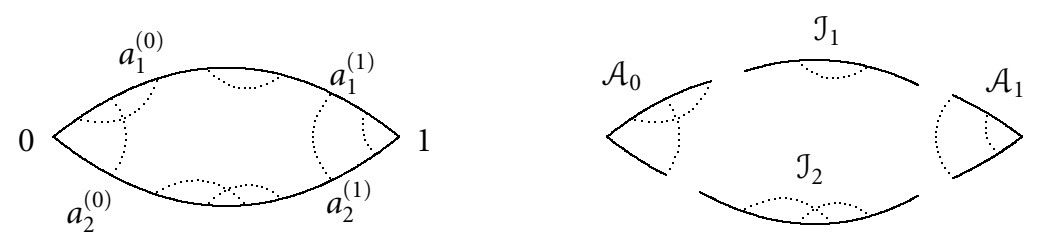

Figure 6: For $i \in\{0,1\}$, the subnetwork $\mathcal{A}_{i}$ associated to $i$ has leaves $a_{1}^{(i)}$ and $a_{2}^{(i)}$. The interval $\mathcal{J}_{e}$ connects $a_{e}^{(0)}$ to $a_{e}^{(1)}$. We use the convention that a bond is drawn to cover the interval on its concave side. 
Next, we introduce concepts and notation that will be used to analyse a single connected component $\left.\Gamma\right|_{\mathcal{A}_{i}}$. Let $\mathcal{A}$ be a network with star shape $\tau$ of degree at most $\Delta$, and let 0 denote the root. We regard $\mathcal{A}$ as a star-shaped network of degree $\Delta$ with some branches possibly having length zero. Since $\mathcal{A}$ is a tree, there is no possible ambiguity in associating an interval to a bond, and hence in defining the notion of a connected graph on $\mathcal{A}$. This natural notion of connectivity is the same as in Definition 2.1, but it applies to a larger class of bonds (all bonds) without reference to the notion of nearness. With this natural notion of connectivity, we define $\mathcal{G}_{\mathcal{A}}^{\text {conn }}$ to be the set of connected graphs on $\mathcal{A}$ consisting of bonds in $\mathcal{B}_{\mathcal{A}}$.

Given $\bar{y} \in \mathbb{Z}^{d \Delta}$, fix $\omega \in \Omega_{\mathcal{A}}(\bar{y})$ (with $y_{i}=0$ if branch $i$ has zero length), so that the leaves of $\mathcal{A}$ are embedded at the components $y_{j} \in \mathbb{Z}^{d}$ of $\bar{y}$. In our application, these $y_{j}$ represent the locations to which the vertices $a_{j}^{(l)}$ defined above are embedded, for some specific $l \in S_{0}$. We must also keep track of the vector $\vec{x}$ of displacements of the embedding of our original network $\mathcal{N}$. These determine the notion of nearness that was used in defining the set $\mathcal{G}_{\mathcal{N}}$ of graphs on the network $\mathcal{N}$, and therefore the notion of nearness is implicitly present in defining a connected component $\mathcal{A}_{i}$. With this in mind, given $\bar{x} \in \mathbb{Z}^{d \Delta}$, we let $\tilde{V}_{\mathcal{A}, \bar{x}}=\tilde{V}_{\mathcal{A}, \bar{x}}(\omega)$ denote the set of bonds $s t, s, t \neq 0$, such that $s \in T_{j}, t \in T_{j^{\prime}}$ for distinct edges $j, j^{\prime}$ of $\tau$ and such that both $s, t$ are near 0 in terms of $\bar{x}$, i.e., $\omega(s) \in B\left(x_{j}\right)$ and $\omega(t) \in B\left(x_{j^{\prime}}\right)$. Note that $\tilde{\mathcal{V}}_{\mathcal{A}, \bar{x}}$ depends on $\bar{x}$ only via the absolute value of the components of $\bar{x}$, as these absolute values define the notion of nearness. We define $\tilde{\mathcal{G}}_{\mathcal{A}, \bar{x}}^{\text {conn }}=\tilde{\mathcal{G}}_{\mathcal{A}, \bar{x}}^{\text {conn }}(\omega) \subset \mathcal{G}_{\mathcal{A}}^{\text {conn }}$ to be the set of connected graphs on $\mathcal{A}$ consisting of bonds in $\tilde{V}_{\mathcal{A}, \bar{x}} \cup \mathcal{H}_{\mathcal{A}}$.

We also define

$$
\pi^{(\Delta)}(\bar{y})=\sum_{\bar{m} \in \mathbb{Z}_{+}^{\Delta}} \sum_{\omega \in \Omega_{\mathcal{A}}(\bar{y})} W_{z_{\mathcal{c}}}(\omega) J_{\mathcal{A}}(\omega), \quad \tilde{\pi}_{\bar{x}}^{(\Delta)}(\bar{y})=\sum_{\bar{m} \in \mathbb{Z}_{+}^{\Delta}} \sum_{\omega \in \Omega_{\mathcal{A}}(\bar{y})} W_{z_{\mathrm{c}}}(\omega) \tilde{J}_{\mathcal{A}, \bar{x}}(\omega),
$$

where the $j$-th component of $\bar{m}$ is the length of the $j$-th branch of $\mathcal{A}$, and $\mathbb{Z}_{+}=$ $\mathbb{N} \cup\{0\}$. The term in (2.12) due to $\bar{m}=\overline{0}$ is $\delta_{\overline{0}, \bar{y}}$, and other terms are zero unless two or more components of $\bar{m}$ are strictly positive.

Given $\nu=(S, E), i \in S_{0}$ and $\vec{x}=\left(x_{1}, \ldots, x_{|E|}\right) \in \mathbb{Z}^{d|E|}$, let $\bar{x}^{(i)}=\left(x_{1}^{(i)}, \ldots, x_{\Delta_{i}}^{(i)}\right)$ be the projection of $\vec{x}$ on $\mathbb{Z}^{d \Delta_{i}}$ defined by $x_{j}^{(i)}=x_{e}$, where $e \in E$ is the $j$-th edge of $\nu$ incident on $i$. Let $|\Delta|=\sum_{i \in S_{0}} \Delta_{i}$.

Proposition 2.2 Using the above notation, and assuming that (2.13) and the sum defining $\tilde{\pi}_{\bar{x}}^{(\Delta)}(\bar{y})$ in $(2.12)$ both converge absolutely,

$$
M_{\nu}(\vec{x})=\sum_{\vec{y} \in \mathbb{Z}^{d}|\Delta|}\left[\prod_{i \in S_{0}} \tilde{\pi}_{\bar{x}^{(i)}}^{\left(\Delta_{i}\right)}\left(\bar{y}^{(i)}\right)\right] \prod_{e \in E} G\left(x_{e}+\delta y_{e}\right),
$$

where $\vec{y}=\left(\bar{y}^{(1)}, \ldots, \bar{y}^{\left(\left|S_{0}\right|\right)}\right)$ and $\delta y_{e}=y_{l}^{\left(e_{2}\right)}-y_{k}^{\left(e_{1}\right)}$, with e the $k$-th edge of $\nu$ incident on $e_{1}$ and the l-th edge of $\nu$ incident on $e_{2}$. 
Proof By (2.7) and (2.10),

$$
M_{\nu}(\vec{x})=\sum_{\vec{n} \in \mathbb{N}|E|} \sum_{\omega \in \Omega_{\mathcal{N}}(\vec{x})} W_{z_{\mathrm{c}}}(\omega) \sum_{\Gamma \in \mathcal{G}_{\mathcal{N}}^{\text {main }}}\left[\prod_{i \in S_{0}}\left[\prod_{\left.b \in \Gamma\right|_{\mathcal{A}_{i}}} U_{b}(\omega)\right]\right] \prod_{e \in E}\left[\prod_{\left.b \in \Gamma\right|_{J_{e}}} U_{b}(\omega)\right] .
$$

We reorganise the summations by fixing the displacements $y_{k}^{(i)}=\omega\left(a_{k}^{(i)}\right)-\omega(i)$ for each $\mathcal{A}_{i}$, and then summing over $\vec{y}=\left(\bar{y}^{(1)}, \ldots, \bar{y}^{\left.|| S_{0} \mid\right)}\right) \in \mathbb{Z}^{d|\Delta|}$. This is allowed by our absolute convergence assumption. Thus (2.14) is equal to

$$
\sum_{\vec{y} \in \mathbb{Z}^{d}|\Delta|} \sum_{\vec{n} \in \mathbb{N}^{|E|}} \sum_{\omega \in \Omega_{\mathcal{N}}(\vec{x})} W_{z_{c}}(\omega) \sum_{\substack{\Gamma \in \mathcal{S}_{\mathcal{N}}^{\text {main }}: \\ \omega\left(a_{k}^{(i)}=\omega(i)+y_{k}^{(i)} \\(\text { all } i, k)\right.}}\left[\prod_{i \in S_{0}}\left[\prod_{b \in \Gamma \mid \mathcal{A}_{i}} U_{b}(\omega)\right]\right] \prod_{e \in E}\left[\prod_{\left.b \in \Gamma\right|_{J_{e}}} U_{b}(\omega)\right] .
$$

We may now perform the summations over $\left.\Gamma\right|_{\mathcal{A}_{i}}$ and $\left.\Gamma\right|_{\mathcal{J}_{e}}$ independently to see that (2.15) is equal to

$$
\begin{aligned}
& \sum_{\vec{y} \in \mathbb{Z}^{d|\Delta|}}\left[\prod_{i \in S_{0}}\left[\sum_{\bar{m} \in \mathbb{Z}_{+}^{\Delta_{i}}} \sum_{\omega \in \Omega_{\mathcal{A}_{i}}\left(\bar{y}^{(i)}\right)} W_{z_{\mathcal{c}}}(\omega) \sum_{\Gamma \in \tilde{\mathcal{S}}_{\mathcal{A}_{i}, \bar{x}^{(i)}}} \prod_{b \in \Gamma} U_{b}(\omega)\right]\right] \\
& \times \prod_{e \in E}\left[\sum_{l \in \mathbb{Z}_{+}} \sum_{\omega \in \Omega_{\mathcal{J}}\left(x_{e}+\delta y_{e}\right)} W_{z_{\mathcal{c}}}(\omega) \sum_{\Gamma \in \mathcal{G}_{\mathcal{J}}} \prod_{b \in \Gamma} U_{b}(\omega)\right],
\end{aligned}
$$

where $\mathcal{A}_{i}$ and $\mathcal{J}$ respectively denote a star-shaped network and an interval, with $|\mathcal{J}|=l$. By $(2.11)$ and (2.12), the quantity in the product over $i$ is $\tilde{\pi}_{\dot{x}^{(i)}}^{\left(\Delta_{i}\right)}\left(\bar{y}^{(i)}\right)$. Since $\sum_{\Gamma \in \mathcal{G}_{\mathcal{J}}} \prod_{b \in \Gamma} U_{b}=\prod_{b \in \mathcal{B}_{\mathcal{J}}}\left[1+U_{b}\right]$, the quantity in the product over $e$ is $G\left(x_{e}+\delta y_{e}\right)$.

\subsection{Proof of Theorem 1.3 Assuming Several Propositions}

In this section, we state several propositions and show that they imply Theorem 1.3. The propositions are proved in Sections 4-9. Each of the propositions requires as hypotheses that $d>4$ and $L \geq L_{0}(d, \nu)$ for some large $L_{0}$, and we do not repeat these hypotheses below.

For $\bar{x}=\left(x_{1}, \ldots, x_{\Delta}\right) \in \mathbb{Z}^{d \Delta}$, we define a multidimensional version of the ball defined in (2.1) by

$$
B(\bar{x})=\left\{\bar{y} \in \mathbb{Z}^{d \Delta}:\left|y_{k}\right| \leq \frac{1}{3}\left|x_{k}\right|(k \in\{1, \ldots, \Delta\})\right\} .
$$

We denote summation over $\bar{y}^{(i)} \in B\left(\bar{x}^{(i)}\right)$ for all $i \in S_{0}$ by $\sum_{\vec{y} \in B(\vec{x})}$. For $x \in \mathbb{Z}^{d}$, we will use the notation

$$
\|x\||=| x \mid \vee 1 .
$$

Although (2.18) does not define a norm on $\mathbb{R}^{d}$, it does obey the triangle inequality. The following proposition gives bounds on $\tilde{\pi}_{\bar{x}}^{(\Delta)}(\bar{y})$. 
Proposition 2.3 There exists $C=C(d, \Delta)<\infty$ such that

$$
\sum_{\bar{y} \in \mathbb{Z}^{d \Delta}: y_{e}=y}\left|\tilde{\pi}_{\bar{x}}^{(\Delta)}(\bar{y})\right| \leq \frac{C}{\|y\|^{2 d-4}} \quad\left(e \in\{1, \ldots, \Delta\}, \bar{x} \in \mathbb{Z}^{d \Delta}\right) .
$$

The bound (2.19) also holds when $\tilde{\pi}_{\bar{x}}^{(\Delta)}(\bar{y})$ is replaced by $\pi^{(\Delta)}(\bar{y})$, and when $J_{\mathcal{A}}$ and $\tilde{J}_{\mathcal{A}, \bar{x}}$ are replaced by their absolute values in (2.12). In addition,

$$
\sum_{\bar{y} \in \mathbb{Z}^{d \Delta}}\left|\tilde{\pi}_{\bar{x}}^{(\Delta)}(\bar{y})\right| \prod_{e=1}^{\Delta} \frac{1}{\left\|y_{e}-w_{e}\right\|^{d-2}} \leq C \prod_{e=1}^{\Delta} \frac{1}{\left\|w_{e} \mid\right\|^{d-2}} \quad\left(\bar{w}, \bar{x} \in \mathbb{Z}^{d \Delta}\right)
$$

$$
\sum_{\bar{y} \notin B(\bar{x})}\left|\tilde{\pi}_{\bar{x}}^{(\Delta)}(\bar{y})\right| \prod_{e=1}^{\Delta} \frac{1}{\left\|y_{e}-x_{e}\right\|^{d-2}} \leq C\left[\prod_{e=1}^{\Delta} \frac{1}{\left\|x_{e}|\||^{d-2}\right.}\right] \sum_{e \in E} \frac{1}{\left\|x_{e}\right\|^{d-4}} \quad\left(\bar{x} \in \mathbb{Z}^{d \Delta}\right) .
$$

For $\Delta \geq 2$, we define

$$
V_{\Delta}=\sum_{\bar{y} \in \mathbb{Z}^{d \Delta}} \pi^{(\Delta)}(\bar{y}), \quad \tilde{V}_{\Delta, \bar{x}}^{\mathrm{in}}=\sum_{\bar{y} \in B(\bar{x})} \tilde{\pi}_{\bar{x}}^{(\Delta)}(\bar{y}),
$$

and $V_{1}=\tilde{V}_{1, \bar{x}}^{\text {in }}=1$. By (2.19) and the remark following (2.19), the series defining $\tilde{V}_{\Delta, \bar{x}}^{\text {in }}$ and $V_{\Delta}$ converge absolutely. Also, the absolute convergence in (2.12) required as a hypothesis in Proposition 2.2 follows. It was shown in [5] that $V_{\Delta}=1+O\left(L^{-d}\right)$ for every $\Delta \geq 2$, and we will give an alternate proof of this fact in Section 6 .

The following five propositions will be used to prove Theorem 1.3.

Proposition 2.4 There exists $C=C(d, \nu)<\infty$ such that

$$
\left|\psi_{\nu}(\vec{x})\right| \leq C\left[\prod_{e \in E} \frac{1}{\left\|x_{e}\right\|^{d-2}}\right] \sum_{i, j \in S: i \neq j} \frac{1}{\left\|v_{i}-v_{j}\right\|^{d-4}} \quad\left(\vec{x} \in \mathbb{Z}^{d|E|}\right) .
$$

Proposition 2.5 Fix $\epsilon_{1}<(d-4) \wedge 1$. There exists $C=C\left(d, \nu, \epsilon_{1}\right)<\infty$ such that

$$
\begin{aligned}
\sum_{\vec{y} \in B(\vec{x})} & {\left[\prod_{i \in S_{0}}\left|\tilde{\pi}_{\bar{x}^{(i)}}^{\left(\Delta_{i}\right)}\left(\bar{y}^{(i)}\right)\right|\right] \sum_{\bar{E} \subset E: \bar{E} \neq \varnothing}\left[\prod_{e \in E \backslash \bar{E}} G\left(x_{e}\right)\right] \prod_{e \in \bar{E}}\left|G\left(x_{e}+\delta y_{e}\right)-G\left(x_{e}\right)\right| } \\
\leq C & {\left[\prod_{e \in E} \frac{1}{\left\|x_{e}\right\|^{d-2}}\right] \sum_{e \in E} \frac{1}{\left.\left\|x_{e}\right\|\right|^{\mid}} \quad\left(\vec{x} \in \mathbb{Z}^{d|E|}\right) . }
\end{aligned}
$$

Proposition 2.6 There exists $C=C(d, \nu)<\infty$ such that

$$
\begin{aligned}
& \sum_{\vec{y} \notin B(\vec{x})} {\left[\prod_{i \in S_{0}}\left|\tilde{\pi}_{\bar{x}^{(i)}}^{\left(\Delta_{i}\right)}\left(\bar{y}^{(i)}\right)\right|\right] \prod_{e \in E} G\left(x_{e}+\delta y_{e}\right) } \\
& \leq C\left[\prod_{e \in E} \frac{1}{\left\|x_{e}\right\|^{d-2}}\right] \sum_{e \in E} \frac{1}{\left\|x_{e} \mid\right\|^{d-4}} \quad\left(\vec{x} \in \mathbb{Z}^{d|E|}\right) .
\end{aligned}
$$


Proposition 2.7 Let $\bar{x}=\left(x_{1}, \ldots, x_{\Delta}\right)$. There exists $C=C(d, \Delta)<\infty$ such that

$$
\left|V_{\Delta}-\tilde{V}_{\Delta, \bar{x}}^{\mathrm{in}}\right| \leq C \sum_{e=1}^{\Delta} \frac{1}{\left\|x_{e}\right\|^{d-4}}
$$

Proposition 2.8 There exists $C=C(d, \nu)<\infty$ such that

$$
\left|\varphi_{\nu}(\vec{x})\right| \leq C\left[\prod_{e \in E} \frac{1}{\left\|x_{e} \mid\right\|^{d-2}}\right] \sum_{e \in E} \frac{1}{\left\|x_{e}\right\|^{\mid 2(d-4)}} \quad\left(\vec{x} \in \mathbb{Z}^{d|E|}\right) .
$$

Propositions 2.4-2.6 are proved in Section 4, using Proposition 2.3 in the proofs of Propositions 2.5-2.6. Proposition 2.3 is proved in Sections 6-7, using the notion of lace introduced in Section 5. Finally, Propositions 2.7 and 2.8 are proved in Sections 8 and 9 , respectively.

Proof of Theorem 1.3 assuming Propositions 2.3-2.8 Recall the decomposition of $G_{\nu}(\vec{x})$ given in (2.9). By Propositions 2.4 and 2.8, $\psi_{\nu}(\vec{x})$ and $\varphi_{\nu}(\vec{x})$ contribute to the error term in (1.11). By Proposition 2.6, the contribution to $M_{\nu}(\vec{x})$ in (2.13) due to the summation over $\vec{y} \notin B(\vec{x})$ is also an error term. The remaining term can be written as

$$
\sum_{\vec{y} \in B(\vec{x})}\left[\prod_{i \in S_{0}} \tilde{\pi}_{\bar{x}^{(i)}}^{\left(\Delta_{i}\right)}\left(\bar{y}^{(i)}\right)\right] \prod_{e \in E}\left[G\left(x_{e}\right)+\left[G\left(x_{e}+\delta y_{e}\right)-G\left(x_{e}\right)\right]\right]
$$

We expand the product over $e$ in (2.28). By Proposition 2.5, the contribution from terms in which one or more factors $\left[G\left(x_{e}+\delta y_{e}\right)-G\left(x_{e}\right)\right]$ occur is an error term. The remaining term is equal to

$$
\prod_{i \in S_{0}} \tilde{V}_{\Delta_{i}, \bar{x}^{(i)}}^{\text {in }} \prod_{e \in E} G\left(x_{e}\right)=\left[\prod_{i \in S_{0}} V_{\Delta_{i}}\right] \prod_{e \in E} G\left(x_{e}\right)-\left[\prod_{i \in S_{0}} V_{\Delta_{i}}-\prod_{i \in S_{0}} \tilde{V}_{\Delta_{i}, \bar{x}^{(i)}}^{\mathrm{in}}\right] \prod_{e \in E} G\left(x_{e}\right) .
$$

By Theorem 1.2, the first term in the right side of (2.29) gives the desired leading asymptotics of $G_{\nu}(\vec{x})$. Using a telescoping representation,

$$
\prod_{i \in S_{0}} V_{\Delta_{i}}-\prod_{i \in S_{0}} \tilde{V}_{\Delta_{i}, \tilde{x}^{(i)}}^{\mathrm{in}}=\sum_{j=1}^{\left|S_{0}\right|}\left[\prod_{i=1}^{j-1} \tilde{V}_{\Delta_{i}, \tilde{x}^{(i)}}^{\mathrm{in}}\right]\left[V_{\Delta_{j}}-\tilde{V}_{\Delta_{j}, \tilde{x}^{(j)}}^{\mathrm{in}}\right]\left[\prod_{i=j+1}^{\left|S_{0}\right|} V_{\Delta_{i}}\right]
$$

(with empty products equal to 1). By Proposition 2.7, and using the bounds on $\tilde{V}_{\Delta_{i,}, \bar{x}^{(j)}}^{\text {in }}$ and $V_{\Delta_{j}}$ that follow from Proposition 2.3, the second term on the right side of (2.29) is therefore also an error term. The above estimates also show that (2.13) converges absolutely. 


\section{Convolution Bounds}

We will make frequent use of the following elementary convolution lemma, whose bounds are depicted diagrammatically in Figure 7.

Lemma 3.1 The following bounds hold for all $z, z_{1}, z_{2}, z_{3}, z_{4} \in \mathbb{Z}^{d}$. For $a \geq b>0$, there exists $C=C(a, b, d)<\infty$ such that

$$
\sum_{w \in \mathbb{Z}^{d}} \frac{1}{\|z-w\|^{a}} \frac{1}{\|w\|^{b}} \leq \begin{cases}C\|z\|^{-b} & (a>d) \\ C\|z\|^{d-a-b} & (a<d<a+b) .\end{cases}
$$

For $d>4$, there exists $C=C(d)<\infty$ such that

$$
\begin{gathered}
\sum_{w \in Z^{d}} \frac{1}{\left\|z_{1}-w\right\|^{2(d-2)}} \frac{1}{\left.\left\|z_{2}-w\right\|\right|^{d-2}} \frac{1}{\left\|z_{3}-w\right\|^{d-2}} \\
\leq C \frac{1}{\left.\left\|z_{2}-z_{1}\right\|\right|^{d-2}} \frac{1}{\left\|z_{3}-z_{1}\right\| \|^{d-2}}, \\
\sum_{w \in \mathbb{Z}^{d}} \frac{1}{\left\|z_{1}-w\right\|^{d-2}} \frac{1}{\left\|z_{2}-w\right\| \|^{d-2}} \frac{1}{\left\|z_{3}-w\right\|^{d-2}} \\
\leq C \frac{1}{\left.\left\|z_{1}-z_{2}\right\|\right|^{d-2}} \sum_{i \neq j} \frac{1}{\left\|z_{i}-z_{j}\right\|^{d-4}}, \\
\sum_{w \in Z^{d}} \frac{1}{\left\|z_{1}-w\right\| \|^{d-2}} \frac{1}{\left\|z_{2}-w\right\|^{d-2}} \frac{1}{\left\|z_{3}-w\right\|^{d-2}} \frac{1}{\left\|z_{4}-w\right\|^{d-2}} \\
\leq C \frac{1}{\left\|z_{1}-z_{2}\right\| \|^{d-2}} \frac{1}{\left.\left\|z_{3}-z_{4}\right\|\right|^{d-2}} \sum_{i \neq j} \frac{1}{\left\|z_{i}-z_{j}\right\|^{d-4}} .
\end{gathered}
$$

Proof The bound (3.1) is proved in [4, Proposition 1.7 (i)]. The bound (3.2) follows from $[4,(4.17)]$, and is also a consequence of the special case $z_{1}=z_{3}$ of (3.4). For (3.3), note that for any $w$ either $\left\|z_{1}-w\right\|\left\|\geq \frac{1}{2}\right\| z_{1}-z_{2} \| \mid$ or $\left\|z_{2}-w\right\| \| \geq$ $\frac{1}{2}\left\|z_{1}-z_{2}\right\|$. . Thus the left side of (3.3) is bounded by

$$
\frac{2^{d-2}}{\left\|z_{1}-z_{2}\right\|^{d-2}} \sum_{w \in \mathbb{Z}^{d}}\left(\frac{1}{\left\|z_{1}-w\right\|^{d-2}}+\frac{1}{\left\|z_{2}-w\right\|^{d-2}}\right) \frac{1}{\left\|z_{3}-w\right\|^{d-2}},
$$

and (3.3) follows from (3.1) with $a=b=d-2$. For (3.4), assume first that

$$
\left\|z_{1}-w\right\| \geq \geq \frac{1}{2}\left\|z_{1}-z_{2}|\|, \quad\|| z_{3}-w\right\|\left\|\geq \frac{1}{2}\right\| z_{3}-z_{4}\|\| .
$$

The contribution to the left side of (3.4) from this case is bounded by

$$
\frac{2^{2(d-2)}}{\left\|z_{1}-z_{2}\right\|^{d-2}\left\|z_{3}-z_{4}\right\|^{d-2}} \sum_{w} \frac{1}{\left\|z_{2}-w\right\|^{d-2}} \frac{1}{\left\|z_{4}-w\right\| \|^{d-2}} .
$$


(a)

$$
0 \longrightarrow z \leq 0 \longleftarrow z
$$

(b)

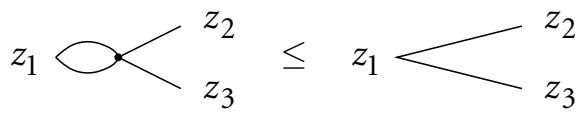

(c)

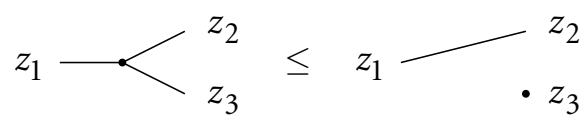

(d)

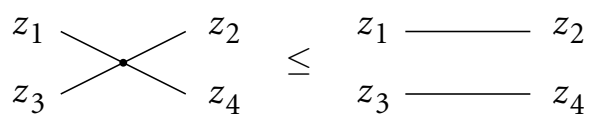

Figure 7: Diagrammatic depiction of (3.1)-(3.4) in (a)-(d) respectively. A diagram line from $w$ to $z$ represents $\|w-z \mid\|^{2-d}$, and the unlabelled vertex $w$ is summed over $\mathbb{Z}^{d}$. For (3.1), we take $a=2 d-4>d$ and $b=d-2$. Factors decaying like $\left\|z_{i}-z_{j} \mid\right\|^{4-d}$ in (3.3)-(3.4) are not shown.

Applying (3.1), this gives a bound of the desired form. There are three similar cases, where one or both of the inequalities in (3.6) is changed to $\left\|z_{2}-w\right\|\left\|\geq \frac{1}{2}\right\| z_{1}-z_{2} \mid \|$ or $\left\|z_{4}-w\right\|\left\|\geq \frac{1}{2}\right\| z_{3}-z_{4} \|$, respectively.

\section{Proof of Propositions 2.4-2.6}

We prove Propositions 2.4-2.6 in Sections 4.1-4.3 respectively. In the remainder of the paper, we use $c$ to denote a finite positive constant which may depend on $d, \epsilon_{1}$ and the shape $\nu$ of the network, but whose exact value is unimportant and may change from line to line.

\subsection{Proof of Proposition 2.4}

Recall the definition of $\psi_{\nu}(\vec{x})$ in (2.4). For $e, e^{\prime} \in E$, we denote by $\mathcal{R}_{\mathcal{N}}^{e, e^{\prime}}$ the set of bonds $s t \in \mathcal{R}_{\mathcal{N}}$ such that $s \in T_{e}$ and $t \in T_{e^{\prime}}$. If the indicator function

$$
1-\prod_{b \in \mathcal{R}_{\mathcal{N}}}\left[1+U_{b}\right]
$$

equals 1 , then there exists at least one $\mathcal{R}_{\mathcal{N}}^{e, e^{\prime}}$ such that

$$
1-\prod_{b \in \mathcal{R}_{\mathcal{N}}^{e, e} f^{\prime}}\left[1+U_{b}\right]=1
$$


Therefore

$$
1-\prod_{b \in \mathcal{R}_{\mathcal{N}}}\left[1+U_{b}\right] \leq \sum_{e, e^{\prime} \in E}\left[1-\prod_{b \in \mathcal{R}_{\mathcal{N}}^{e, e^{\prime}}}\left[1+U_{b}\right]\right]
$$

Using $1+U_{b} \leq 1$ to estimate the product over $\mathcal{V}_{\mathcal{N}}$ in $(2.4)$, we obtain

$$
\left|\psi_{\nu}(\vec{x})\right| \leq \sum_{e, e^{\prime} \in E} \sum_{\vec{n} \in \mathbb{N}^{|E|}} \sum_{\omega \in \Omega_{\mathcal{N}}(\vec{x})} W_{z_{\mathcal{c}}}(\omega)\left[\prod_{b \in \mathcal{H}_{\mathcal{N}}}\left[1+U_{b}(\omega)\right]\right]\left[1-\prod_{b \in \mathcal{R}_{\mathcal{N}}^{e, e^{\prime}}}\left[1+U_{b}(\omega)\right]\right] .
$$

The factor $\prod_{b \in \mathcal{H}_{\mathcal{N}}}\left[1+U_{b}\right]$ equals 1 if and only if the embedded path corresponding to each edge of $\nu$ is a self-avoiding walk. When $e=e^{\prime}=\left(e_{1}, e_{2}\right)$, the only bond in $\mathcal{R}_{\mathcal{N}}^{e, e}$ is the bond $e_{1} e_{2}$, and hence $1-\prod_{b \in \mathcal{R}_{\mathcal{N}}^{e, e}}\left[1+U_{b}\right]$ is equal to $-U_{e_{1} e_{2}}=$ $\mathbb{1}_{\left[\omega\left(e_{1}\right)=\omega\left(e_{2}\right)\right]}=\mathbb{1}_{\left[v_{e_{1}}=v_{e_{2}}\right]}$. This does not contribute to the behavior of $\psi_{\nu}(\vec{x})$ in the limit we are concerned with, in which $\left|v_{e_{1}}-v_{e_{2}}\right| \rightarrow \infty$. We may therefore restrict the summation over $e, e^{\prime}$ in (4.2) to $e \neq e^{\prime}$. When $e \neq e^{\prime}$, the factor $1-\prod_{b \in \mathcal{R}_{\mathcal{N}}^{e, e^{\prime}}}\left[1+U_{b}\right]$ equals 1 only when the embedded path from $v_{e_{1}}$ to $v_{e_{2}}$ intersects the embedded path from $v_{e_{1}^{\prime}}$ to $v_{e_{2}^{\prime}}$ at some $w \in \mathbb{Z}^{d}$. Moreover, by definition of $\mathcal{R}_{\mathcal{N}}$, this $w$ must lie in the set $R_{e, e^{\prime}}$, defined below, and hence the right side of (4.2) is bounded above by

$$
\sum_{e, e^{\prime} \in E: e \neq e^{\prime}}\left[\prod_{f \neq e, e^{\prime}} G\left(x_{f}\right)\right] \sum_{w \in R_{e, e^{\prime}}} G\left(v_{e_{1}}-w\right) G\left(w-v_{e_{2}}\right) G\left(v_{e_{1}^{\prime}}-w\right) G\left(w-v_{e_{2}^{\prime}}\right) .
$$

Recall the definition of $B_{v}(x)$ from (2.1).

(i) If $e$ and $e^{\prime}$ are not adjacent, then $R_{e, e^{\prime}}=\mathbb{Z}^{d}$;

(ii) if $e$ and $e^{\prime}$ are adjacent at a unique vertex $i \in S_{0}$, then $R_{e, e^{\prime}}=B_{v_{i}}\left(x_{e}\right)^{\mathrm{c}} \cup B_{v_{i}}\left(x_{e^{\prime}}\right)^{\mathrm{c}}$;

(iii) if $e$ and $e^{\prime}$ both have the same endvertices $i, j \in S_{0}$, then $\left|x_{e}\right|=\left|x_{e^{\prime}}\right|$ and $R_{e, e^{\prime}}=$ $B_{v_{i}}\left(x_{e}\right)^{\mathrm{c}} \cap B_{v_{j}}\left(x_{e}\right)^{\mathrm{c}}$.

Recall from (1.10) that $G(y) \leq c\|\| y \|^{2-d}$. The contribution to (4.3) due to case (i) gives a bound of the desired form, by (3.4). In case (ii), if $w \in B_{v_{e_{1}}}\left(x_{e}\right)^{\text {c }}$ we use the bound $G\left(v_{e_{1}}-w\right) \leq c \mid\left\|x_{e}\right\| \|^{2-d}$ and apply (3.3), and similarly for the other contribution to this case. In case (iii), we extract two factors $\left\|\left|x_{e} \|\right|^{2-d}\right.$ as in case (ii), and then apply (3.1) to bound the remaining convolution. (Case (iii) corresponds to the bottom picture in Figure 4.)

\subsection{Proof of Proposition 2.5}

We first consider the product $\left[\prod_{e \in E \backslash \bar{E}} G\left(x_{e}\right)\right] \prod_{e \in \bar{E}}\left|G\left(x_{e}+\delta y_{e}\right)-G\left(x_{e}\right)\right|$, for a fixed nonempty subset $\bar{E} \subset E$. Fix $f \in \bar{E}$. The first product can be estimated using the upper bound of Theorem 1.2. For the second product, since $\vec{y} \in B(\vec{x})$ we have $\left|x_{e}+\delta y_{e}\right| \geq \frac{1}{3}\left|x_{e}\right|>0$ and thus $\left|G\left(x_{e}+\delta y_{e}\right)-G\left(x_{e}\right)\right| \leq\left|G\left(x_{e}+\delta y_{e}\right)\right|+\left|G\left(x_{e}\right)\right| \leq$ $c\left|x_{e}\right|^{2-d}$. Applying this bound for all $e \neq f$ gives

$$
\left[\prod_{e \in E \backslash \bar{E}} G\left(x_{e}\right)\right] \prod_{e \in \bar{E}}\left|G\left(x_{e}+\delta y_{e}\right)-G\left(x_{e}\right)\right| \leq c\left|G\left(x_{f}+\delta y_{f}\right)-G\left(x_{f}\right)\right| \prod_{e \in E: e \neq f}\left|x_{e}\right|^{2-d}
$$


Recall from $(1.8)$ that $\epsilon_{2}=2(d-4) \wedge 2$. We will show below that

$$
\left|G\left(x_{f}+\delta y_{f}\right)-G\left(x_{f}\right)\right| \leq c\left|x_{f}\right|^{1-d}\left(\left|\delta y_{f}\right|+\left|x_{f}\right|^{1-\epsilon_{2}+\alpha}\right),
$$

with $\alpha>0$ arbitrarily small. It therefore suffices to show that

$$
\left|x_{f}\right|^{-1} \sum_{\vec{y} \in B(\vec{x})}\left[\prod_{i \in S_{0}}\left|\tilde{\pi}_{\bar{x}^{(i)}}^{\left(\Delta_{i}\right)}\left(\bar{y}^{(i)}\right)\right|\right]\left(\left|\delta y_{f}\right|+\left|x_{f}\right|^{1-\epsilon_{2}+\alpha}\right) \leq c\left|x_{f}\right|^{-\epsilon_{1}} .
$$

But by (2.19), the left side of (4.6) is bounded above by

$$
c\left|x_{f}\right|^{-1} \sum_{y, y^{\prime} \in B\left(x_{f}\right)} \frac{\left|y^{\prime}-y\right|+\left|x_{f}\right|^{1-\epsilon_{2}+\alpha}}{\left\|\left|\|\|^{2 d-4}\right|\right\| y^{\prime} \mid \|^{2 d-4}} \leq c^{\prime}\left|x_{f}\right|^{-1} \sum_{y \in B\left(x_{f}\right)} \frac{|y|+\left|x_{f}\right|^{1-\epsilon_{2}+\alpha}}{\left.\|y\|\right|^{2 d-4}}
$$

which gives (4.6).

It remains to prove (4.5). By (1.9) and the inequality $|x+\delta y| \geq \frac{1}{3}|x|>0$,

$$
G(x+\delta y)-G(x)=A \sigma^{-2}\left[|x+\delta y|^{2-d}-|x|^{2-d}\right]+O\left(|x|^{2-d-\epsilon_{2}+\alpha}\right) .
$$

By Taylor's theorem, the quantity in brackets equals

$$
\frac{d}{d s}\left[\frac{1}{(s|x+\delta y|+(1-s)|x|)^{d-2}}\right]_{s=s_{0}}=\frac{-(d-2)(|x+\delta y|-|x|)}{\left(s_{0}|x+\delta y|+\left(1-s_{0}\right)|x|\right)^{d-1}},
$$

for some $s_{0} \in(0,1)$. Since $|x+\delta y| \geq \frac{1}{3}|x|$, the denominator is bounded below by $c^{-1}|x|^{d-1}$, and since ||$x+\delta y|-| x|| \leq|\delta y|$, the absolute value of (4.9) is bounded above by $c|x|^{1-d}|\delta y|$. This proves (4.5).

\subsection{Proof of Proposition 2.6}

The left side of (2.25) is bounded by the sum over $j \in S_{0}$ of

$$
\sum_{\substack{\vec{y} \in \mathbb{Z}^{d|\Delta|} \\ \bar{y}^{(j)} \notin B\left(\bar{x}^{(j)}\right)}}\left[\prod_{i \in S_{0}}\left|\tilde{\pi}_{\bar{x}^{(i)}}^{\left(\Delta_{i}\right)}\left(\bar{y}^{(i)}\right)\right|\right] \prod_{e \in E} \frac{c}{\left.\left\|\left|x_{e}+\delta y_{e}\right|\right\|\right|^{d-2}} .
$$

Given $j \in S_{0}$, we order the set $S_{0} \backslash\{j\}$ in an arbitrary but fixed manner. We perform the sums over $\bar{y}^{(i)}$ for $i \in S_{0} \backslash\{j\}$ in this order, using (2.20). For a given $i$, we sum the factor $\left|\tilde{\pi}_{\bar{x}^{(i)}}^{\left(\Delta_{i}\right)}\left(\bar{y}^{(i)}\right)\right|$ together with any factors $\left\|\mid x_{e}^{(i)}+\delta y_{e}^{(i)}\right\| \|^{2-d}$ or $\left.\left\|x_{e}^{(i)}-y_{e}^{(i)}\right\|\right|^{2-d}$, with $e$ incident on $i$, depending on whether or not the other endpoint of $e$ has already been taken into account. Letting $E(j)$ denote the edges of $\nu$ that are incident on $j$, and applying (2.21), it follows that (4.10) is bounded above by

$$
\begin{aligned}
& \prod_{e^{\prime} \in E \backslash E(j)} \frac{c}{\left\|x_{e^{\prime}}|\||^{d-2}\right.} \sum_{\bar{y}^{(j)} \notin B\left(\bar{x}^{(j)}\right)}\left|\tilde{\pi}_{\bar{x}^{(j)}}^{\left(\Delta_{j}\right)}\left(\bar{y}^{(j)}\right)\right| \prod_{e \in E(j)} \frac{1}{\left\|x_{e}^{(j)}-y_{e}^{(j)}\right\|^{d-2}} \\
& \leq c\left[\prod_{e \in E} \frac{1}{\left\|x_{e}\right\|^{d-2}}\right] \sum_{e \in E(j)} \frac{1}{\left\|x_{e}\right\|^{d-4}} .
\end{aligned}
$$




\section{The Lace Expansion}

The lace expansion was introduced by Brydges and Spencer [1] to analyse weakly self-avoiding walks indexed by a "time" interval, for $d>4$. In [5], the expansion was extended from an expansion on an interval to an expansion on a tree. In this section, we recall from [5] the definitions and properties that we will require. Throughout this section, we restrict attention to a star-shaped network $\mathcal{A}=\mathcal{A}\left(\tau_{\Delta}, \bar{m}\right)$, rooted at the branch point 0 of degree $\Delta \geq 2$ and with leaves $a_{1}, \ldots, a_{\Delta}$. The path in $\mathcal{A}$ from 0 to $a_{e}$ has length $m_{e}$, and will be referred to as branch $T_{e}$. We assume in this section that $m_{e}>0$ for all $e$. We will use the content of this section to prove Propositions 2.3, 2.7 and 2.8 .

\subsection{Laces and Resummation}

Definition 5.1 Given a connected graph $\Gamma$ on $\mathcal{A}$ (see Definition 2.1) and a branch $T_{e}$, let $\Gamma_{e}=\Gamma_{e}(\Gamma)$ denote the set of bonds st covering the branch point 0 such that either $s$ or $t$ is in $T_{e}$, say $\Gamma_{e}=\left\{s_{1} t_{1}, \ldots, s_{l} t_{l}\right\}$ with each $s_{k} \notin T_{e}, t_{k} \in T_{e}$. From $\Gamma_{e}$, we select the element or elements for which the distance from $t_{k}$ to 0 is maximal. If there is a unique such bond, then we say it is the bond of $\Gamma$ that is associated to branch $T_{e}$. If there is more than one such bond in $\Gamma_{e}$, then we select from those with $t_{k}$ maximally distant from 0 the one with $s_{k}$ furthest from 0 . If this still does not specify a unique bond, then we choose the $s_{k}$ that lies on the branch with the highest label, and refer to $s_{k} t_{k}$ as the bond associated to branch $T_{e}$. We denote the bond associated to $T_{e}$ by $b^{(e)}$.

Definition 5.2 A lace on $\mathcal{A}$ is a connected graph $L$ such that:

1. if $b \in L$ covers the branch point, then it is associated to branch $T_{e}$ for some $e$;

2. if $b \in L$ does not cover the branch point, then $L \backslash\{b\}$ is not connected.

We denote by $\mathcal{L}_{\mathcal{A}}$ the laces consisting of bonds in $\mathcal{B}_{\mathcal{A}}$, and by $\tilde{\mathcal{L}}_{\mathcal{A}, \bar{x}}=\tilde{\mathcal{L}}_{\mathcal{A}, \bar{x}}(\omega) \subset \mathcal{L}_{\mathcal{A}}$ the set of laces on $\mathcal{A}$ consisting of bonds in $\tilde{\mathcal{V}}_{\mathcal{A}, \bar{x}} \cup \mathcal{H}_{\mathcal{A}}$.

Definitions 5.1-5.2 correspond to [5, Definitions 2.3-2.4]. The following prescription, which associates to a connected graph $\Gamma$ on $\mathcal{A}$ a lace $L \subset \Gamma$, was introduced in [5, Section 2.3]. Given a connected graph $\Gamma$ and a branch $T_{e}$, we first construct a lace $\mathrm{L}_{\Gamma}(e)=\left\{s_{1} t_{1}, \ldots, s_{l} t_{l}\right\}$ on an interval in $\mathcal{A}$ that contains $T_{e}$, according to the $T_{e}$-lace construction defined as follows. First we choose $s_{1} t_{1}$ as $b^{(e)}$ with $s_{1} \notin T_{e}$, $t_{1} \in T_{e}$. If $t_{1} \neq a_{e}$, then we determine $t_{2}, s_{2}, \ldots$ by

$$
t_{k}=\max \left\{t: \exists s<t_{k-1} \text { such that } s t \in \Gamma\right\}, \quad s_{k}=\min \left\{s: s t_{k} \in \Gamma\right\},
$$

where the order implied by the max and min is the order on $T_{e}$ obtained by identifying $T_{e}$ with the interval $\left[0, m_{e}\right]$ ( 0 is the branch point of degree $\Delta$ ). The procedure terminates as soon as $t_{l}=a_{e}$. The prescription that associates to a connected graph $\Gamma$ a lace $L_{\Gamma}$ is then given by

$$
\mathrm{L}_{\Gamma}=\bigcup_{e=1}^{\Delta} \mathrm{L}_{\Gamma}(e)
$$


By [5, Proposition 2.5], $\mathrm{L}_{\Gamma}$ is a lace.

Given a lace $L \in \mathcal{L}_{\mathcal{A}}$, we define $\mathcal{C}_{\mathcal{A}}(L)$ to be the set of bonds $b \in \mathcal{B}_{\mathcal{A}} \backslash L$ such that $\mathrm{L}_{L \cup b}=L$. Similarly, given $L \in \tilde{\mathcal{L}}_{\mathcal{A}, \bar{x}}$ (or $L \in \mathcal{L}_{\mathcal{A}}$ ), we define $\tilde{\mathcal{C}}_{\mathcal{A}, \bar{x}}(L)$ to be the set of bonds $b \in \tilde{V}_{\mathcal{A}, \bar{x}} \cup \mathcal{H}_{\mathcal{A}} \backslash L$ such that $\mathrm{L}_{L \cup b}=L$. Bonds in $\mathcal{C}_{\mathcal{A}}(L)$ or $\tilde{\mathcal{C}}_{\mathcal{A}, \bar{x}}(L)$ are said to be compatible with $L$. It follows from [5, Proposition 2.6] that for $\Gamma \in \mathcal{G}_{\mathcal{A}}^{\text {conn }}, \mathrm{L}_{\Gamma}=L$ if and only if $L \subset \Gamma$ is a lace in $\mathcal{L}_{\mathcal{A}}$ and $\Gamma \backslash L \subset \mathcal{C}_{\mathcal{A}}(L)$. Similarly, given $\Gamma \in \tilde{\mathcal{G}}_{\mathcal{A}, \bar{x}}^{\text {conn }}$, $\mathrm{L}_{\Gamma}=L$ if and only if $L \subset \Gamma$ is a lace in $\tilde{\mathcal{L}}_{\mathcal{A}, \bar{x}}$ and $\Gamma \backslash L \subset \tilde{\mathcal{C}}_{\mathcal{A}, \bar{x}}(L)$. Recalling (2.11), this leads, as in [5, Section 2.4], to the resummation identities

$$
J_{\mathcal{A}}=\sum_{L \in \mathcal{L}_{\mathcal{A}}}\left[\prod_{b \in L} U_{b}\right] \prod_{b \in \mathcal{C}_{\mathcal{A}}(L)}\left[1+U_{b}\right], \quad \tilde{J}_{\mathcal{A}, \bar{x}}=\sum_{L \in \tilde{\mathcal{L}}_{\mathcal{A}, \bar{x}}}\left[\prod_{b \in L} U_{b}\right] \prod_{b \in \tilde{\mathcal{E}}_{\mathcal{A}, \bar{x}}(L)}\left[1+U_{b}\right]
$$

For $N \geq 1$, let $\tilde{L}_{\mathcal{A}, \bar{x}}^{(N)} \subset \tilde{\mathcal{L}}_{\mathcal{A}, \bar{x}}$ and $\mathcal{L}_{\mathcal{A}}^{(N)} \subset \mathcal{L}_{\mathcal{A}}$ denote the sets of laces on $\mathcal{A}$ consisting of exactly $N$ bonds. We define

$$
\begin{aligned}
J_{\mathcal{A}}^{(N)} & =\sum_{L \in \mathcal{L}_{\mathcal{A}}^{(N)}}\left[\prod_{b \in L}\left[-U_{b}\right]\right] \prod_{b \in \mathcal{C}_{\mathcal{A}}(L)}\left[1+U_{b}\right], \\
\tilde{J}_{\mathcal{A}, \bar{x}}^{(N)} & =\sum_{L \in \tilde{\mathcal{L}}_{\mathcal{A}, \bar{x}}^{(N)}}\left[\prod_{b \in L}\left[-U_{b}\right]\right] \prod_{b \in \tilde{\mathcal{C}}_{\mathcal{A}, \bar{x}}(L)}\left[1+U_{b}\right] .
\end{aligned}
$$

Both quantities in (5.3) are non-negative, by definition.

\subsection{Classification and Properties of Laces}

We recall the classification of laces, and some related lemmas, from [5]. Further details can be found in [5, Section 3].

Definition 5.3 Let $\mathcal{A}=\mathcal{A}\left(\tau_{\Delta}, \bar{m}\right)$ with $\Delta \geq 2$.

(a) A lace $L \in \mathcal{L}_{\mathcal{A}}$ is reducible if there is a proper subset $F \subset\{1, \ldots, \Delta\}$ such that $L$ can be written as a disjoint union of laces on each of $\bigcup_{e \in F} T_{e}$ and $\bigcup_{e \in F^{c}} T_{e}$. An irreducible lace is a lace that is not reducible.

(b) A lace is cyclic if it is irreducible and its bonds covering the branch point can be ordered as $\left\{i_{k} j_{k}: k=1, \ldots, \Delta\right\}$, with $j_{k}$ and $i_{k+1}$ on the same branch for $1 \leq k \leq \Delta$. By convention, $i_{\Delta+1}=i_{1}$.

(c) An irreducible lace that is not cyclic is called acyclic.

A lace $L$ uniquely determines a partition $I_{1}, \ldots, I_{k}$ of $\{1, \ldots, \Delta\}$ into subsets of cardinality at least 2 , such that $L$ is the disjoint union of the irreducible laces obtained by restricting $L$ to $\bigcup_{e \in I_{j}} T_{e}$. We refer to these irreducible laces as the irreducible components of $L$.

Definition 5.4 A connected graph (in particular, a lace) is called minimal if removal of any of its bonds results in a graph that is not connected. A lace that is not minimal is called non-minimal. 
Although it is possible for a lace to be non-minimal, any minimal connected graph is a lace.

Lemma 5.5 ([5, Lemma 3.7]) Let $\mathcal{A}=\mathcal{A}\left(\tau_{\Delta}, \bar{m}\right)$ with $\Delta \geq 3$ and suppose $L \in \mathcal{L}_{\mathcal{A}}$ is acyclic. Then there is a branch $T_{a}$ such that there is only one bond covering the branch point 0 with an endpoint in $T_{a}$, and such that the restriction of $L$ to $\bigcup_{e \neq a} T_{e}$ is a lace on $\bigcup_{e \neq a} T_{e}$.

Lemma 5.6 ([5, Lemma 3.12]) Let $\mathcal{A}=\mathcal{A}\left(\tau_{\Delta}, \bar{m}\right)$ with $\Delta \geq 2$. Let $L$ be a nonminimal cyclic lace on $\mathcal{A}$, and let $i, j \in \mathcal{A}$ be such that $L \backslash\{i j\} \in \mathcal{L}_{\mathcal{A}}$. Then $L \backslash\{i j\}$ is an acyclic lace on $\mathcal{A}$.

\section{Proof of Proposition 2.3}

In this section, we state a bound on $\tilde{\pi}$ in Proposition 6.1 and use this bound to prove Proposition 2.3. The proof of Proposition 6.1 will be given in Section 7.

The statement of the bound requires several definitions. We recall (5.3), and for $N \geq 1$ we define

$$
\pi_{N}^{(\Delta)}(\bar{y})=\sum_{\bar{m} \in \mathbb{Z}_{+}^{\Delta}} \sum_{\omega \in \Omega_{\mathcal{A}}(\bar{y})} W_{z_{\mathcal{c}}}(\omega) J_{\mathcal{A}}^{(N)}, \quad \tilde{\pi}_{\bar{x}, N}^{(\Delta)}(\bar{y})=\sum_{\bar{m} \in \mathbb{Z}_{+}^{\Delta}} \sum_{\omega \in \Omega_{\mathcal{A}}(\bar{y})} W_{z_{\mathrm{c}}}(\omega) \tilde{J}_{\mathcal{A}, \bar{x}}^{(N)}(\omega) .
$$

We also define $\pi_{0}^{(\Delta)}(\bar{y})=\tilde{\pi}_{\bar{x}, 0}^{(\Delta)}(\bar{y})=\delta_{\overline{0}, \bar{y}}$, so that

$$
\pi^{(\Delta)}(\bar{y})=\sum_{N=0}^{\infty}(-1)^{N} \pi_{N}^{(\Delta)}(\bar{y}), \quad \tilde{\pi}_{\bar{x}}^{(\Delta)}(\bar{y})=\sum_{N=0}^{\infty}(-1)^{N} \tilde{\pi}_{\bar{x}, N}^{(\Delta)}(\bar{y}) .
$$

In the sum over laces implicit in $J_{\mathcal{A}}^{(N)}$ or $\tilde{J}_{\mathcal{A}, \bar{x}}^{(N)}$, branches of $\mathcal{A}$ with zero length (for which $\left.m_{e}=0\right)$ are ignored. Thus laces on star shapes of degree $2, \ldots, \Delta$ contribute to (6.1), depending on the number of non-zero components of $\bar{m}$ (which must be at least two for $N \geq 1$ ). Let

$$
\Pi_{N}^{(\Delta)}(\bar{y})=\sum_{\bar{m} \in \mathbb{N}^{\Delta}} \sum_{\omega \in \Omega_{\mathcal{A}}(\bar{y})} W_{z_{\mathrm{c}}}(\omega) J_{\mathcal{A}}^{(N)}, \quad \tilde{\Pi}_{\bar{x}, N}^{(\Delta)}(\bar{y})=\sum_{\bar{m} \in \mathbb{N}^{\Delta}} \sum_{\omega \in \Omega_{\mathcal{A}}(\bar{y})} W_{z_{\mathrm{c}}}(\omega) \tilde{J}_{\mathcal{A}, \bar{x}}^{(N)}(\omega)
$$

denote the restriction of (6.1) to the case where all $\Delta$ branches have positive length. For $I \subset\{1, \ldots, \Delta\}$, let $\bar{y}_{I}$ denote the vector whose components are $y_{j}$ with $j \in I$. For $N \geq 1, \tilde{\pi}_{\bar{x}, N}^{(\Delta)}(\bar{y})$ is a sum of terms of the form $\delta_{\overline{0}, \bar{y}_{I}} \tilde{\Pi}_{\bar{x}, N}^{(I I \mid)}\left(\bar{y}_{I}\right)$ over subsets $I \subset$ $\{1, \ldots, \Delta\}$ of cardinality $2 \leq|I| \leq \Delta$, and similarly for $\pi_{N}^{(\Delta)}(\bar{y})$. 
For $\Delta \geq 2$, we write $z_{\Delta+1}=z_{1}$ and define

$$
\begin{array}{r}
C^{(\Delta)}(\bar{y}, \bar{z})=\prod_{j=1}^{\Delta} \frac{1}{\left\|z_{j}\right\|^{d-2}} \frac{1}{\left\|z_{j+1}-y_{j}\right\|^{d-2}} \frac{1}{\left\|y_{j}-z_{j}\right\|^{2(d-2)}}, \\
\tilde{C}^{(2)}\left(y_{1}, y_{2}, z_{1}, z_{2}\right)=\delta_{z_{1}, z_{2}} \prod_{j=1}^{2} \frac{1}{\left.\left\|y_{j}\right\|\right|^{d-2}} \frac{1}{\left\|y_{j}-z_{1}\right\|^{2(d-2)}}, \\
P^{(\Delta)}(\bar{y}, \bar{z})= \begin{cases}C^{(\Delta)}(\bar{y}, \bar{z}) & (\Delta \geq 3) \\
C^{(2)}(\bar{y}, \bar{z})+\tilde{C}^{(2)}(\bar{y}, \bar{z}) & (\Delta=2) .\end{cases}
\end{array}
$$

Given $2 \leq \Delta^{\prime}<\Delta$, and given a mapping $u$ with domain $\left\{\Delta^{\prime}+1, \ldots, \Delta\right\}$ and with $u(j) \in\left\{0, y_{1}, \ldots, y_{j-1}, z_{1}, \ldots, z_{j-1}\right\}$, let

$$
Q_{u, j}(\bar{y}, \bar{z})=\frac{1}{\left\|y_{j}\right\|^{d-2}} \frac{1}{\left\|y_{j}-z_{j}\right\|^{2(d-2)}} \frac{1}{\left\|z_{j}-u(j)\right\| \|^{d-2}} .
$$

Finally, for $\Delta \geq 2$, we define

$$
B^{(\Delta)}(\bar{y})=\sum_{\Delta^{\prime}=2}^{\Delta} \sum_{\bar{z} \in B(\bar{x})} \sum_{u} P^{\left(\Delta^{\prime}\right)}(\bar{y}, \bar{z}) \prod_{j=\Delta^{\prime}+1}^{\Delta} Q_{u, j}(\bar{y}, \bar{z}),
$$

where the third sum is over all mappings $u$ of the type indicated above, and where $P^{\left(\Delta^{\prime}\right)}(\bar{y}, \bar{z})$ depends only on the first $\Delta^{\prime}$ components of $\bar{y}$ and $\bar{z}$. Diagrammatic representations of $B^{(2)}(\bar{y})$ and $B^{(3)}(\bar{y})$ are given in Figure 8 . For $B^{(3)}(\bar{y})$, the first diagram is the $\Delta^{\prime}=3$ term of (6.8), and the remaining nine terms arise from the $\Delta^{\prime}=2$ term, with five choices for $u(3)$ for $C^{(2)}$ and four choices for $\tilde{C}^{(2)}$.

For $I \subset\{1, \ldots, \Delta\}$, we write $\Sigma_{I}$ for the set of permutations of $I$. Given $p \in \Sigma_{I}$, we let $p\left(\bar{y}_{I}\right)$ denote the vector with components $y_{p(j)}, j \in I$. We write $\beta=L^{\alpha-2}$ for the small factor appearing on the right side of (1.10).

Proposition 6.1 Let $d>4$ and $\Delta \geq 2$. Fix $\bar{y} \in \mathbb{Z}^{d \Delta}$. There are constants $C$ and $L_{0}$ (depending on $d$ and $\Delta$ ) such that for $L \geq L_{0}$,

$$
\sum_{N=1}^{\infty} \tilde{\Pi}_{\bar{x}, N}^{(\Delta)}(\bar{y}) \leq C \beta^{\Delta} \sum_{I_{1}, \ldots, I_{k}} \prod_{j=1}^{k} \sum_{p_{j} \in \Sigma_{I_{j}}} B^{\left(\left|I_{j}\right|\right)}\left(p_{j}\left(\bar{y}_{I_{j}}\right)\right),
$$

where the first sum on the right side is over all partitions of $\{1, \ldots, \Delta\}$ into subsets $I_{1}, \ldots, I_{k}$ of cardinality at least 2 . The same bound holds for $\Pi_{N}^{(\Delta)}(\bar{y})$, with the sum over $\bar{z}$ in (6.8) extended from $B(\bar{x})$ to $\mathbb{Z}^{d \Delta}$.

Under the hypotheses of Proposition 6.1, and in view of the relationship between $\tilde{\pi}_{\bar{x}, N}^{(\Delta)}$ and $\tilde{\Pi}_{\bar{x}, N}^{(\Delta)}$ described below $(6.3)$, the sum $\tilde{\pi}_{\bar{x}}^{(\Delta)}(\bar{y})=\sum_{N=0}^{\infty}(-1)^{N} \tilde{\pi}_{\bar{x}, N}^{(\Delta)}(\bar{y})$ obeys the bound

$$
\left|\tilde{\pi}_{\bar{x}}^{(\Delta)}(\bar{y})\right| \leq \delta_{\overline{0}, \bar{y}}+C \beta^{2} \sum_{I_{1}, \ldots, I_{k}} \delta_{\overline{0}_{,} \bar{y}_{i^{c}}} \prod_{j=1}^{k} \sum_{p_{j} \in \Sigma_{I_{j}}} B^{\left(\left|I_{j}\right|\right)}\left(p_{j}\left(\bar{y}_{I_{j}}\right)\right) .
$$




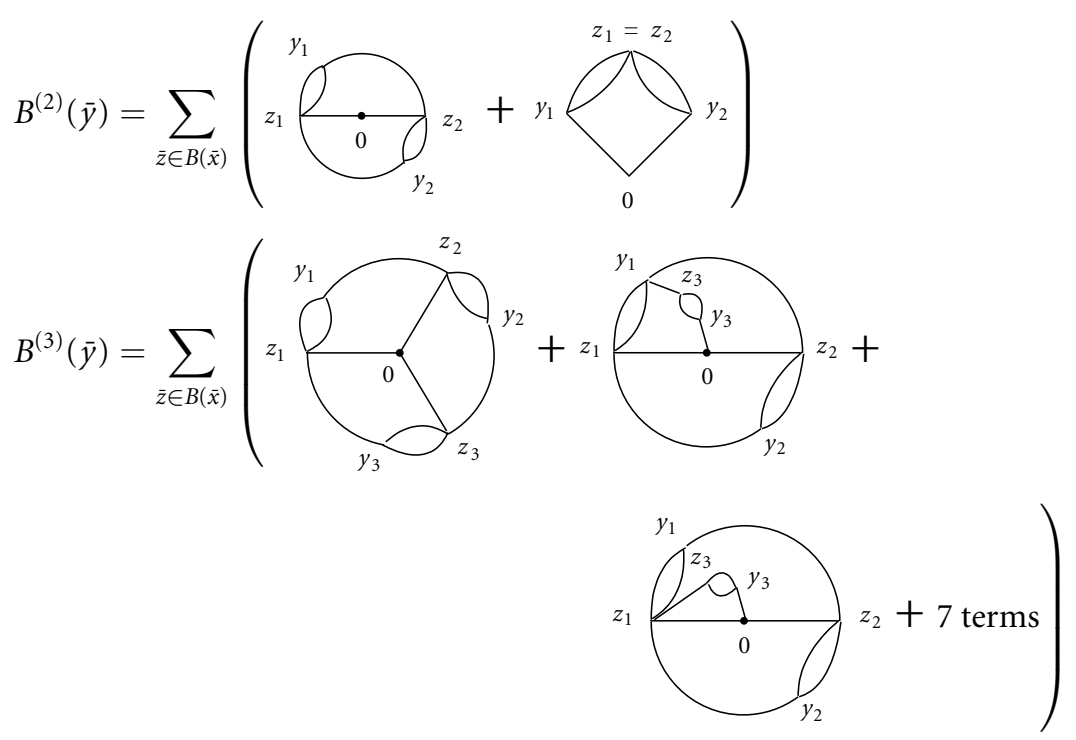

Figure 8: Diagrammatic representations of $B^{(2)}(\bar{y})$ and $B^{(3)}(\bar{y})$.

In (6.10), the sum over $I_{1}, \ldots, I_{k}$ is a sum over disjoint subsets of $\{1, \ldots, \Delta\}$, each of cardinality at least 2 , whose union $\hat{I}$ may be smaller than $\{1, \ldots, \Delta\}$. The same bound holds for $\pi^{(\Delta)}(\bar{y})$, with the sum over $\bar{z}$ in (6.8) extended from $B(\bar{x})$ to $\mathbb{Z}^{d \Delta}$.

\section{Proof of Proposition 2.3 assuming Proposition 6.1}

Proof of (2.19) We deduce (2.19) from (6.10), and the corresponding statement for $\pi^{(\Delta)}(\bar{y})$ will follow from the comment below (6.10). This will automatically prove the absolute convergence statement given under (2.19). In fact, using $J_{\mathcal{A}}^{(N)}$ and $\tilde{J}_{\mathcal{A}, \bar{x}}^{(N)}$ in (6.3) and summing over $N$ in (6.9) gives an upper bound on the sums in (2.12) when $J_{\mathcal{A}}$ and $\tilde{J}_{\mathcal{A}, \bar{x}}$ are replaced by their absolute values.

We analyse the factor $\sum_{p \in \Sigma_{I}} B^{(|I|)}\left(p\left(\bar{y}_{I}\right)\right)$. For this we relabel $\bar{y}_{I}$ as $y_{1}, \ldots, y_{|I|}$ for convenience. We will prove

$$
\sum_{\bar{y} \in \mathbb{Z}^{d|I|}: y_{e}=y} \sum_{p \in \Sigma_{I}} B^{(|I|)}(p(\bar{y})) \leq \frac{c}{\|y\| \|^{2 d-4}}, \quad \sum_{\bar{y} \in \mathbb{Z}^{d|I|}} \sum_{p \in \Sigma_{I}} B^{(|I|)}(p(\bar{y})) \leq c,
$$

for any $e \in I$. This suffices by the following argument. Given the index $e$ and $y$ in (2.19) first assume that $y \neq 0$. Then only terms with $e \in \hat{I}=I_{1} \cup \cdots \cup I_{k}$ contribute to (6.10). We apply the first inequality of (6.11) to the unique $I_{j}$ such that $e \in I_{j}$, and apply the second one otherwise, to get (2.19). If $y=0$, we also have terms with $e \notin \hat{I}$, and for these we only need the second inequality in (6.11).

The second inequality in (6.11) follows from the first one, since the bound is summable. In proving the first inequality it is enough to consider terms where $p$ 
is the identity. Indeed, if we can prove the bound in (6.11) without summation over permutations and for any $e \in I$, (6.11) follows as well. To analyse $B^{(|I|)}(\bar{y})$ recall (6.8). Let $2 \leq \Delta^{\prime} \leq|I|$, and let $K(\bar{y}, \bar{z})$ denote a general term from (6.8) with $\Delta=|I|$, that is

$$
K(\bar{y}, \bar{z})=P^{\left(\Delta^{\prime}\right)}(\bar{y}, \bar{z}) \prod_{j=\Delta^{\prime}+1}^{|I|} Q_{u, j}(\bar{y}, \bar{z}) .
$$

We will prove that

$$
\sum_{\bar{y}, \bar{z} \in Z^{d|| \mid}: y_{e}=y} K(\bar{y}, \bar{z}) \leq \frac{c}{\|y\|^{2 d-4}}, \quad \sum_{\bar{y}, \bar{z} \in Z^{d|| I}::_{e}=z} K(\bar{y}, \bar{z}) \leq \frac{c}{\|z\|^{2 d-4}},
$$

for any $e \in I$ and for any mapping $u$. This is sufficient for our claim on the sum of $B^{(I I)}(\bar{y})$. In fact, the first inequality of (6.13) is sufficient, but it will be convenient for the proof to have the second one as well.

To prove (6.13), we consider first the case $\Delta^{\prime}=|I|$, so that $K(\bar{y}, \bar{z})=P^{(|I|)}(\bar{y}, \bar{z})$. The proof for this case is by induction on $|I|$. We begin the induction with $|I|=2$, and consider $P^{(2)}=C^{(2)}+\tilde{C}^{(2)}$. Recall the diagrams in the first line of Figure 8. We will make frequent use of (3.1)-(3.4), and the reader is encouraged to draw the diagrams involved and make use of the pictures in (a)-(d) of Figure 7. The cases when $y_{1}$ or $y_{2}$ is kept fixed are symmetric, and similarly for $z_{1}$ and $z_{2}$. To bound the sum over $y_{2}, z_{1}, z_{2}$ of $C^{(2)}$, we proceed as follows. First, we use (3.2) to perform the sums over $z_{1}$ and $z_{2}$. This gives a bound of the form

$$
\frac{c}{\left.\left\|y_{1}\right\|\right|^{d-2}} \sum_{y_{2} \in \mathbb{Z}^{d}} \frac{1}{\left\|y_{2}\right\|^{d-2}} \frac{1}{\left\|y_{2}-y_{1}\right\|^{2 d-4}} \leq \frac{c}{\left\|y_{1}\right\|^{2 d-4}},
$$

where (3.1) was applied with $a=2 d-4>d$ and $b=d-2$ in (6.14). To bound the sum over $y_{1}, y_{2}, z_{2}$ of $C^{(2)}$ we use (3.1) to perform the sums over $y_{1}$ and $y_{2}$. This leaves an expression similar to the left side of (6.14) with $y_{1}$ and $y_{2}$ replaced by $z_{1}$ and $z_{2}$. This can be easily seen from the diagrams as well. From here we proceed as before. For $\tilde{C}^{(2)}$ first consider the case when $y_{1}$ is fixed. We bound the sum over $y_{2}$ using (3.1), and then bound the resulting sum over $z_{1}$ using (3.1) again. This gives an upper bound of the form $\left\|y_{1}\right\| \|^{4-2 d}$, as required. When $z_{1}$ is fixed, we apply (3.1) twice to bound the sums over $y_{1}$ and $y_{2}$, which yields an upper bound $\left\|z_{1}\right\|{ }^{4-2 d}$, as desired.

To advance the induction, for the case $\Delta^{\prime}=|I|$, we may assume by symmetry that $e \neq|I|$. We use (3.2) to perform the sum over $z_{|I|}$. The part involving $y_{|I|}$ becomes

$$
\sum_{y_{|I|} \in \mathbb{Z}^{d}} \frac{1}{\left\|y_{|I|}-y_{|I|-1} \mid\right\|^{d-2}} \frac{1}{\|\left. y_{|I|}||\right|^{d-2}} \frac{1}{\left.\left\|y_{|I|}-z_{1}\right\|\right|^{d-2}} \leq \frac{c}{\left.\left\|y_{|I|-1}-z_{1}\right\|\right|^{d-2}},
$$

by (3.3). This gives rise to $P^{(|I|-1)}$, and the desired estimate then follows from the induction hypothesis. This completes the inductive proof of the case $\Delta^{\prime}=|I|$. 
Consider now the general case $\Delta^{\prime} \leq|I|$. The proof for this case is by induction on the value of $|I|-\Delta^{\prime}$. The base case $|I|=\Delta^{\prime}$ has just been proved. Assume $|I|>\Delta^{\prime}$. Then separating the last $Q_{u, j}$ factor appearing in $K(\bar{y}, \bar{z})$ we can write

$$
K(\bar{y}, \bar{z})=K_{0}(\bar{y}, \bar{z}) Q_{u,|I|}(\bar{y}, \bar{z}),
$$

where

$$
K_{0}(\bar{y}, \bar{z})=P^{\left(\Delta^{\prime}\right)}(\bar{y}, \bar{z}) \prod_{j=\Delta^{\prime}+1}^{|I|-1} Q_{u, j}(\bar{y}, \bar{z}) .
$$

Note that $K_{0}(\bar{y}, \bar{z})$ depends only on the first $|I|-1$ components of $\bar{y}$ and $\bar{z}$, and by the induction hypothesis $K_{0}(\bar{y}, \bar{z})$ satisfies (6.13). We distinguish between the cases $e<|I|$ and $e=|I|$. In the first case we bound $\sum_{y_{|I|}, z_{|I|}} Q_{u,|I|}(\bar{y}, \bar{z})$ by a constant using (3.1) first with $a=2 d-4, b=d-2$ and then with $a=b=d-2$. Then we use (6.13) for $K_{0}$. In the case $e=|I|$ we distinguish between the subcases $u(|I|)=0$ and $u(|I|) \neq 0$. In the first subcase we bound the sum of $K_{0}(\bar{y}, \bar{z})$ over all of its variables (which factors from the remaining sum) by a constant, using that the bounds in (6.13) are summable. We bound the remaining sum over $y_{|I|}$ or $z_{|I|}$ using (3.1). In the subcase $u(|I|) \neq 0$ we bound the sum of $K_{0}(\bar{y}, \bar{z})$ with the variable $u(|I|)$ fixed by $c\|u(|I|)\| \|^{4-2 d}$ using the induction hypothesis. The product of this bound with $Q_{u,|I|}(\bar{y}, \bar{z})$ can be estimated using two applications of (3.1).

In the above analysis, the sum over $\bar{z}$ in (6.8) has been extended from $B(\bar{x})$ to all of $\mathbb{Z}^{d|I|}$. Since (6.10) holds also for $\pi_{N}^{(\Delta)}(\bar{y})$ with this extension of the sum over $\bar{z}$, this proves (2.19) also for $\pi^{(\Delta)}(\bar{y})$.

Proof of (2.20) The proof is a modification of the proof of (2.19), and we indicate the necessary changes. Again it is sufficient to prove that for any expression $K(\bar{y}, \bar{z})$ in (6.12) we have

$$
\sum_{\bar{y}, \bar{z} \in \mathbb{Z}^{d|I|}} K(\bar{y}, \bar{z}) \prod_{e=1}^{|I|} \frac{1}{\left\|y_{e}-w_{e}\right\|^{d-2}} \leq c \prod_{e=1}^{|I|} \frac{1}{\left\|w_{e}\right\|^{d-2}} .
$$

In the language of diagrams, there is an extra "arm" reaching out from each $y_{e}$ to a fixed vertex $w_{e}$. Consider the sum over $y_{1}, y_{2}, z_{1}, z_{2}$ of $C^{(2)}$ with the extra factors $\left.\left\|y_{1}-w_{1}\right\|\right|^{2-d}\left\|y_{2}-w_{2}\right\|^{2-d}$. Application of (3.2) to the sums over $z_{1}$ and $z_{2}$ gives an upper bound

$$
\sum_{y_{1}, y_{2} \in \mathbb{Z}^{d}} \frac{1}{\left\|y_{1}-y_{2}\right\|^{2 d-4}} \frac{1}{\left\|y_{1}\right\| \|^{d-2}} \frac{1}{\left.\left\|y_{2}\right\|\right|^{d-2}} \frac{1}{\left.\left\|y_{1}-w_{1}\right\|\right|^{d-2}} \frac{1}{\left\|y_{2}-w_{2}\right\|^{d-2}} .
$$

This gives the desired estimate $c\left\|\mid w_{1}\right\|\left\|^{2-d}\right\| w_{2} \|^{2-d}$, after two other applications of (3.2). In the case of $\tilde{C}^{(2)}$ we also arrive at (6.19) after summing over $z_{1}$ and using (3.4). When $3 \leq \Delta^{\prime}=|I|$ we perform the sum over $z_{|I|}$ as before. In (6.15), an extra 
factor $\left\|y_{|I|}-w_{|I|} \mid\right\|^{2-d}$ is now present, and an application of (3.4) gives an upper bound $\left\|y_{|I|-1}-z_{1}\left|\left\|^{2-d}\right\|\right| w_{|I|}||^{2-d}\right.$. This allows us to use induction on $|I|$ as before. For the case $\Delta^{\prime}<|I|$ we again use (6.16) and induction. Application of (3.2) and (3.3) gives the bound

$$
\sum_{y_{|I|}, z_{|I|}} Q_{u,|I|}(\bar{y}, \bar{z}) \frac{1}{\left\|y_{|I|}-w_{|I|} \mid\right\|^{d-2}} \leq \frac{c}{\left\|w_{|I|}\right\|^{d-2}},
$$

and we get the desired bound by applying $(6.18)$ to $K_{0}(\bar{y}, \bar{z})$.

Proof of (2.21) For the first time in the proof, we make use of the fact that the summation over $\bar{z}$ is restricted to $B(\bar{x})$ in (6.8), rather than a full sum over all of $\mathbb{Z}^{d \Delta}$. The proof is again a modification of the proof of (2.19). It is enough to prove

$$
\sum_{\substack{\bar{y} \in \mathbb{Z}^{d|I|}, \bar{z} \in B(\bar{x}) \\ y_{e} \notin B\left(x_{e}\right)}} K(\bar{y}, \bar{z}) \prod_{j=1}^{|I|} \frac{1}{\left\|y_{j}-x_{j}\right\|^{d-2}} \leq c\left[\prod_{j=1}^{|I|} \frac{1}{\left\|x_{j}\right\|^{d-2}}\right] \frac{1}{\left\|x_{e}\right\| \|^{d-4}}
$$

for any $e \in I$. For the case of $C^{(2)}$ we bound

$$
\sum_{y_{1} \notin B\left(x_{1}\right)} \sum_{y_{2} \in \mathbb{Z}^{d}} \sum_{z_{1} \in B\left(x_{1}\right)} \sum_{z_{2} \in B\left(x_{2}\right)} C^{(2)}\left(y_{1}, y_{2}, z_{1}, z_{2}\right) \frac{1}{\left\|x_{1}-y_{1}\right\| \|^{d-2}} \frac{1}{\left\|x_{2}-y_{2}\right\|^{d-2}}
$$

as follows. We perform the sum over $y_{2}$ using (3.2), and bound the resulting factor $\left\|x_{2}-z_{2}\right\|^{2-d}$ by $c\left\|x_{2}\right\| \|^{2-d}$ using $z_{2} \in B\left(x_{2}\right)$. We then bound the sums over $z_{1}$ and $z_{2}$ using (3.2) and (3.1), respectively. The result is an upper bound of the form

$$
\begin{aligned}
& \frac{c}{\left\|x_{2}\right\|^{d-2}} \sum_{y_{1} \notin B\left(x_{1}\right)} \frac{1}{\left\|y_{1}\right\|^{2 d-4}} \frac{1}{\left\|x_{1}-y_{1}\right\|^{d-2}} \\
& \quad \leq \frac{c}{\left\|x_{2}\right\|^{d-2}} \frac{1}{\left.\left\|x_{1}\right\|\right|^{d-2}} \sum_{y_{1}} \frac{1}{\left\|y_{1}\right\|^{d-2}} \frac{1}{\left\|x_{1}-y_{1}\right\|^{d-2}} .
\end{aligned}
$$

By (3.1), the sum on the right side is bounded by $c\left\|x_{1}\right\| \|^{4-d}$. Similarly, for $\tilde{C}^{(2)}$ we first perform the sum over $y_{2}$, and bound $\left\|x_{2}-z_{1}\right\| \|^{2-d}$ by $\left.c\left\|x_{2}\right\|\right|^{2-d}$ using $z_{1}=z_{2} \in$ $B\left(x_{2}\right)$. Then applying (3.1) to the sum over $z_{1}$ we arrive at (6.23) again.

The case $3 \leq \Delta^{\prime}=|I|$ is handled by induction on $|I|$. By symmetry we may assume $e \neq|I|$. We handle the extra factor $\left\|y_{|I|}-x_{|I|}\right\|^{2-d}$ arising in (6.15) as we did in the proof of (2.20), and extract the extra decay $\left\|x_{e}\right\|^{4-d}$ using the induction hypothesis.

In the case $\Delta^{\prime}<|I|$ we again use induction on $|I|-\Delta^{\prime}$ and (6.16). We distinguish between the cases $e<|I|$ and $e=|I|$. If $e<|I|$, we apply (6.20) with $w_{|I|}$ replaced by $x_{|I|}$, giving a factor $\left\|x_{|I|} \mid\right\|^{2-d}$. The remaining sums are estimated using (6.21) 
for $K_{0}(\bar{y}, \bar{z})$, giving the extra decay $\left\|x_{e}\right\| \|^{4-d}$. If $e=|I|$, we extract the extra decay $\left\|\left|x_{|I|}\right|\right\|^{4-d}$ from

$$
\sum_{y_{|I|} \notin B\left(x_{|I|}\right), z|I|} Q_{\left.u, \mid x_{|I|}\right)}(\bar{y}, \bar{z}) \frac{1}{\| y_{|I|}-x_{|I|}||^{d-2}}
$$

as follows. We bound $\left\|y_{|I|} \mid\right\|^{2-d}$ appearing in $Q_{u,|I|}(\bar{y}, \bar{z})$ from above by $c \| x_{|I|}||^{2-d}$, using $y_{|I|} \notin B\left(x_{|I|}\right)$. Using (3.1) to bound the sum over $y_{|I|}$, for the rest of the expression we get the upper bound

$$
\begin{aligned}
& \sum_{z_{|I|} \in B\left(x_{|I|}\right)} \frac{1}{\left\|z_{|I|}-x_{|I|}|\||^{d-2}\right.} \frac{1}{\left.\left\|z_{|I|}-u(|I|)\right\|\right|^{d-2}} \\
& \leq \frac{c}{\| x_{|I|}||^{d-2}} \sum_{z_{|I|} \in B\left(x_{|I|}\right)} \frac{1}{\left.\left\|z_{|I|}-u(|I|)\right\|\right|^{d-2}},
\end{aligned}
$$

where $z_{|I|} \in B\left(x_{|I|}\right)$ was used in (6.24). The sum on the right side of (6.24) is bounded by a multiple of $\left\|x_{|I|} \mid\right\|^{2}$, uniformly in $u(|I|)$, leading to an upper bound of the desired form $c\left|\left\|x_{|I|} \mid\right\|^{2-d}\left\|x_{|I|}\right\|^{4-d}\right.$. Application of (6.18) to $K_{0}(\bar{y}, \bar{z})$ with $\bar{x}$ replacing $\bar{w}$ completes the proof.

\section{Proof of Proposition 6.1}

In this section, we prove Proposition 6.1. Our starting point is the expression

$$
\tilde{\Pi}_{\bar{x}, N}^{(\Delta)}(\bar{y})=\sum_{\bar{m} \in \mathbb{N}^{\Delta}} \sum_{\omega \in \Omega_{\mathcal{A}}(\bar{y})} W_{z_{\mathcal{c}}}(\omega) \sum_{L \in \tilde{\mathcal{L}}_{\mathcal{A}, \bar{x}}^{(N)}}\left[\prod_{b \in L}\left[-U_{b}\right]\right] \prod_{b \in \tilde{\mathcal{C}}_{\mathcal{A}, \bar{x}}(L)}\left[1+U_{b}\right],
$$

which follows from (5.3) and (6.3). Here $\mathcal{A}$ is the star-shaped tree with branches of length $m_{1}, \ldots, m_{\Delta}$, all of which are strictly positive.

\subsection{Bound on $\tilde{\Pi}$ in Terms of Diagrams}

The set $\tilde{\mathcal{L}}_{\mathcal{A}, \bar{x}}^{(N)}$ depends on the embedding $\omega$, whereas the set $\mathcal{L}_{\mathcal{A}}^{(N)}$ does not. For $L \in$ $\mathcal{L}_{\mathcal{A}}^{(N)}$, let $\mathbb{1}_{[L]}(\omega)$ equal 1 if $L \in \tilde{\mathcal{L}}_{\mathcal{A}, \bar{x}}^{(N)}(\omega)$ and equal 0 otherwise. Then (7.1) can be rewritten as

$$
\tilde{\Pi}_{\bar{x}, N}^{(\Delta)}(\bar{y})=\sum_{\bar{m} \in \mathbb{N}^{\Delta} \Delta} \sum_{L \in \mathcal{L}_{\mathcal{A}}^{(N)}} \sum_{\omega \in \Omega_{\mathcal{A}}(\bar{y})} W_{z_{c}}(\omega) \mathbb{1}_{[L]}(\omega)\left[\prod_{b \in L}\left[-U_{b}\right]\right] \prod_{b \in \tilde{e}_{\mathcal{e}, \tilde{x}}(L)}\left[1+U_{b}\right] .
$$

Given $L$, the factor $\prod_{b \in L}\left[-U_{b}\right]$ is nonzero only if $\omega(s)=\omega(t)$ for every st $\in L$. If $s t \in L$ covers the branch point 0 with $s$ on branch $e$ and $t$ on branch $f$, then $\mathbb{1}_{[L]}(\omega)$ is nonzero only if the additional restriction $\omega(s)=\omega(t) \in B\left(x_{e}\right) \cap B\left(x_{f}\right)$ applies.

Let $L=\left\{s_{1} t_{1}, \ldots, s_{N} t_{N}\right\}$ be a lace on $\mathcal{A}$ consisting of $N$ bonds. The vertices $s_{i}$ and $t_{i}$, together with the branch point 0 , determine $2 N$ intervals $I_{1}, \ldots, I_{2 N}$ on the 
tree $\mathcal{A}$, some of which may have length 0 . We group laces according to their "shape". We say that laces $L$ and $L^{\prime}$ have the same shape if they only differ in the length of the intervals $I_{j}$, and the order of the vertices $s_{i}$ and $t_{i}$ on each branch is the same in $L$ and $L^{\prime}$. Having the same shape does not quite define an equivalence relation on laces (transitivity may fail in some cases). However this will only lead later to some harmless overcounting.

We regard $\omega$ as consisting of a collection of embeddings $\omega^{(j)}$ of the intervals $I_{j}$. Note that $W_{z_{\mathrm{c}}}(\omega)$ factors into the product of $W_{z_{\mathrm{c}}}\left(\omega^{(j)}\right)$ over the intervals. For each interval $I_{j}$, all bonds $b \in \mathcal{B}_{I_{j}}$ are compatible with $L$. This implies that each $\omega^{(j)}$ must be self-avoiding in order to contribute to the right hand side of (7.2). We obtain an upper bound for (7.2) by replacing $\left[1+U_{b}\right]$ by 1 for all other bonds compatible with $L$.

It is then standard to obtain an upper bound on $\tilde{\Pi}_{\bar{x}, N}^{(\Delta)}(\bar{y})$ in terms of Feynman diagrams, where laces having different shapes give rise to different diagrams. An example is depicted in Figure 9. Diagram vertices have degree 4 , except 0 has degree $\Delta$ and each $y_{e}$ has degree 3 . The value $A(\mathcal{F})$ of a diagram $\mathcal{F}$ is determined as follows. Given a shape, we let $\epsilon_{j}=1$ when the length $\left|I_{j}\right|$ of the $j$-th interval is strictly positive for all laces with this shape, and $\epsilon_{j}=0$ otherwise. Thus $\epsilon_{j}$ depends on a shape. A diagram line arising from interval $I_{j}$ and joining vertices $u, v$ corresponds to $H_{j}(u-v)$, where

$$
H_{j}(x)=G(x)-\epsilon_{j} \delta_{x, 0} .
$$

The resulting product of factors $H_{j}$ is then summed over all unlabelled vertices of $\mathcal{F}$. Vertices corresponding to bond endpoints for a bond that does not cover the origin are summed over $\mathbb{Z}^{d}$, whereas a vertex corresponding to bond endpoints for a bond with endpoints on $T_{e}^{\circ}$ and $T_{f}^{\circ}$, with $e \neq f$, is summed over $B\left(x_{e}\right) \cap B\left(x_{f}\right)$. The sum is the value $A(\mathcal{F})$ of $\mathcal{F}$. Since

$$
H_{j}(x) \leq \frac{C \beta^{\epsilon_{j}}}{\|x\|^{d-2}}
$$

by (1.10), for an upper bound we will regard the diagram line arising from an interval $I_{j}$ and joining vertices $u, v$ as $C \beta^{\epsilon_{j}}\|u-v\|^{2-d}$. Let $\tilde{\mathcal{D}}_{N}(\bar{y})$ denote the set of possible diagrams arising from $N$-bond laces, where the tilde denotes the restriction on vertex locations for vertices that correspond to lace bonds that cover 0 . Then

$$
\tilde{\Pi}_{\bar{x}, N}^{(\Delta)}(\bar{y}) \leq \sum_{\mathcal{F} \in \tilde{\mathcal{D}}_{N}(\bar{y})} A(\mathcal{F})
$$

The number of distinct diagrams in $\tilde{\mathcal{D}}_{N}(\bar{y})$ is bounded above by $C^{N}$ for some $C=C(\Delta)$. Also, it is not difficult to see that each lace bond that does not cover the branch point gives rise to at least one nonzero interval, so that $\sum_{j=1}^{2 N} \epsilon_{j}$ is bounded below by $N$ - const. Thus we obtain an exponentially small factor in each term in (7.5), due to the factors $\beta$ in (7.4). This factor compensates for growing factors $C^{N}$ due to the number of diagrams and due to constants that occur in bounding each 

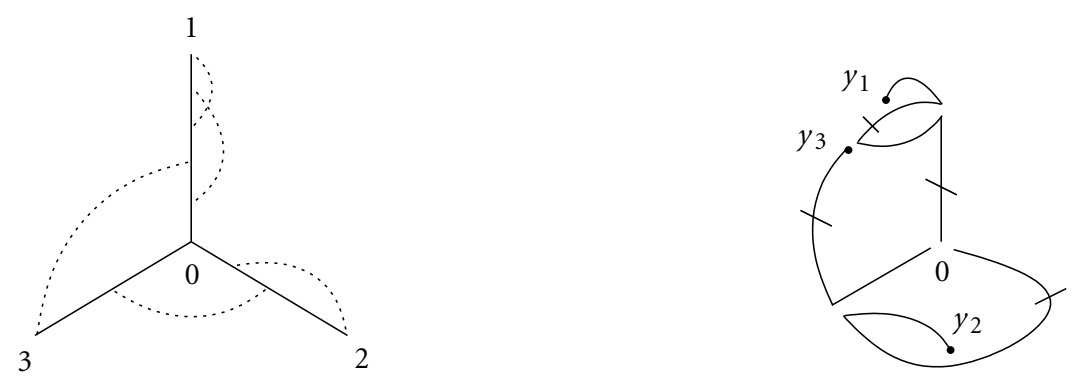

Figure 9: A lace shape and corresponding Feynman diagram $\mathcal{F}$. Slashed diagram lines may have zero length.

line by $c\|u-v\| \|^{2-d}$, and permits the summation over $N$ to be performed. Since $\sum_{j=1}^{2 N} \epsilon_{j} \geq \Delta$, it leads to the correct power $\beta^{\Delta}$ in (6.9). We therefore take $\beta=1$ in what follows, since we are guaranteed by the above to obtain the necessary factors of $\beta$.

\subsection{Reduction of Diagrams}

In this section, we show how it is possible to estimate diagrams in terms of a smaller number of basic diagrams.

Given a lace $L$ and a branch $e$, let $N^{(e)}$ denote the number of bonds of $L$ that have both endpoints on branch $e$. Suppose that $N^{(e)} \geq 2$. Then the bonds $i j$ and $k l$ on branch $e$ that are closest to the leaf of branch $e$ are ordered as $i<k<j<l$, with $l$ the leaf. Let $L^{\prime}$ denote the connected graph obtained by replacing $i j$ and $k l$ by the single bond $i l$. It is not difficult to verify that $L^{\prime}$ is a lace. Let $\mathcal{F} \in \tilde{\mathcal{D}}_{N}(\bar{y})$ denote the diagram corresponding to $L$, and let $\mathcal{F}^{\prime} \in \tilde{\mathcal{D}}_{N-1}(\bar{y})$ denote the diagram corresponding to $L^{\prime}$. We claim that there is a constant $C$, depending only on $d$, such that

$$
A(\mathcal{F}) \leq C A\left(\mathcal{F}^{\prime}\right) .
$$

To prove (7.6), we note that $\omega(k)=\omega(l)=y_{e}$. Since $L$ is a lace, $j$ is the only endpoint of a bond in $L$ that is covered by $k l$. Let $w_{1}=\omega(j)$ denote the vertex in $\mathcal{F}$ corresponding to $j$. Let $w_{2}$ and $w_{3}$ denote the diagram vertices, other than $y_{e}$, that are adjacent to $w_{1}$ in $\mathcal{F}$. Using (3.2) to perform the summation over $w_{1}$ in $A(\mathcal{F})$, we obtain

$$
\begin{gathered}
\sum_{w_{1}} \frac{1}{\left\|w_{2}-w_{1}\right\| \|^{d-2}} \frac{1}{\left\|w_{3}-w_{1}\right\|^{d-2}} \frac{1}{\left\|w_{1}-y_{e}\right\|^{2(d-2)}} \\
\leq \frac{c}{\left\|w_{2}-y_{e}\right\|^{d-2}} \frac{1}{\left\|w_{3}-y_{e}\right\|^{d-2}} .
\end{gathered}
$$

See Figure 10. This replaces part of the diagram for $\mathcal{F}$ by the corresponding part for $\mathcal{F}^{\prime}$, which proves (7.6). 


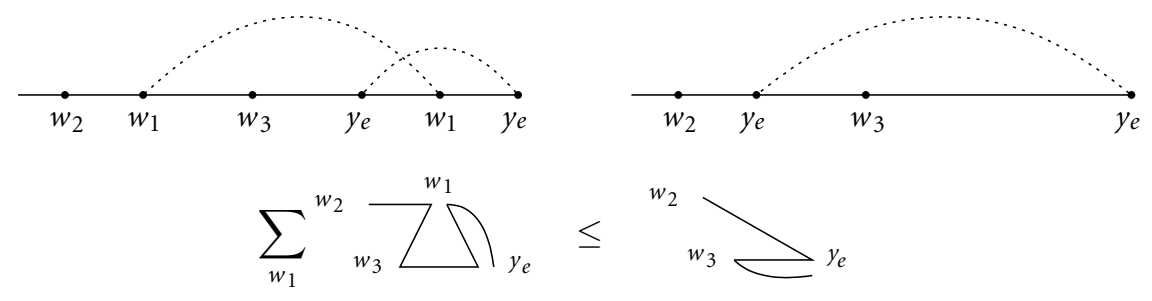

Figure 10: Illustration of the bound (7.7). Vertex labels on the upper figure represent the lattice sites in $\mathbb{Z}^{d}$ to which the vertices are embedded.

If we begin with a diagram in $\tilde{\mathcal{D}}_{N}(\bar{y})$ that has $N^{(e)} \geq 1$ for each $e$, we can use (7.6) repeatedly to reduce the diagram to $C^{N}$ times a diagram having $N^{(e)}=1$ for each $e$. For convenience, we will also show that a diagram with $N^{(e)}=0$ for some $e$ can be bounded by a diagram with $N^{(e)}=1$ for all $e$. Given a lace with $N^{(e)}=0$ and with leaf $j$ on branch $e$, assume that $j$ is not an endpoint of any other lace bond. The case in which $j$ is an endpoint of another lace bond or bonds is a degenerate case that arises from taking an interval or intervals to have zero length in the case we are assuming. We add a new bond $k l$ to the lace in such a way that $k l$ covers $j$, and covers no other endpoint of a lace bond, so that $l$ becomes the new leaf. Since we may now allow all intervals $I_{j}$ to have zero length (because we have already extracted the necessary factors $\beta$ ), the diagram with $N^{(e)}=0$ is just a term in the diagram with $N^{(e)}=1$ in which $w_{1}=y_{e}$, in the notation of the previous paragraph. Thus the diagram with $N^{(e)}=0$ is bounded above by the diagram with $N^{(e)}=1$.

This reduces the estimation of all diagrams to estimation of diagrams having $N^{(e)}=1$ for all $e$. We call such diagrams basic diagrams. The number of diagrams in $\tilde{\mathcal{D}}_{N}(\bar{y})$ that reduce to a given basic diagram is bounded by $C^{N}$.

\subsection{Bounds on Basic Diagrams}

Let $\tilde{\mathcal{D}}^{\text {basic }}(\bar{y})$ denote the set of basic diagrams, arising from laces with $N^{(e)}=1$ for each $e$. In view of the above analysis, to prove Proposition 6.1 it suffices to prove the following proposition.

Proposition 7.1 The sum of basic diagrams, with 0 of degree $\Delta \geq 2$, is bounded by

$$
\sum_{\mathcal{F} \in \tilde{\mathcal{D}}_{\text {basic }}(\bar{y})} A(\mathcal{F}) \leq c \sum_{I_{1}, \ldots, I_{k}} \prod_{j=1}^{k} \sum_{p_{j} \in \Sigma_{I_{j}}} B^{\left(\left|I_{j}\right|\right)}\left(p_{j}\left(\bar{y}_{I_{j}}\right)\right),
$$

where $I_{1}, \ldots, I_{k}$ denotes a partition of $\{1, \ldots, \Delta\}$ into subsets of cardinality at least 2 .

Proof Recall the classification of laces in Section 5.2. We only consider laces for which $N^{(e)}=1$ for each $e$. Also, it suffices to consider only irreducible laces, since 
the diagram corresponding to a reducible lace factors into subdiagrams corresponding to the irreducible components of the reducible lace. The sum over $I_{1}, \ldots, I_{k}$ in (7.8) represents a sum over irreducible components. Thus we are concerned in what follows only with $k=1$ and $I=I_{1}=\{1, \ldots, \Delta\}$.

Fix $\Delta \geq 2$. We give separate arguments according to whether a basic diagram corresponds to an irreducible lace that is minimal cyclic, acyclic, or non-minimal cyclic.

Case 1: Acyclic Laces with $\Delta=2$ The basic diagram corresponding to this case has value $\sum_{z \in B\left(x_{1}\right) \cap B\left(x_{2}\right)} \tilde{C}^{(2)}\left(y_{1}, y_{2}, z, z\right)$.

Case 2: Minimal Cyclic Laces Suppose that $L$ is a minimal cyclic lace. Then, up to a relabelling of the variables, the basic diagram corresponding to $L$ has value $C^{(\Delta)}(\bar{y}, \bar{z})$, with $\bar{z}$ summed over $z_{j} \in B\left(x_{j}\right) \cap B\left(x_{j-1}\right)$. For an upper bound, we relax this to $\bar{z} \in B(\bar{x})$. Taking into account the possible relabellings using permutations, and also taking into account Case 1 if $\Delta=2$, this gives the $\Delta^{\prime}=\Delta$ term of the expression (6.8) for $B^{(\Delta)}(\bar{y})$.

Case 3: Non-Minimal Cyclic Laces with $\Delta=2$ Fix $\Delta=2$. Suppose that $L$ is a non-minimal basic cyclic lace, and that the bond $b$ associated to branch 2 can be removed to yield a lace $\tilde{L}$. We claim that the value of the diagram corresponding to $L$ is bounded by a constant multiple of the value of the diagram corresponding to $\tilde{L}$. To prove this claim, let $w$ be the diagram variable associated to the endpoints of $b$, and let $w_{1}, w_{2}$ and $w_{3}, w_{4}$ be the diagram variables corresponding to the lace bond endpoints adjacent to the two endpoints of $b$. By (3.4),

$$
\sum_{w} \prod_{i=1}^{4} \frac{1}{\left\|w-w_{i}\right\|^{d-2}} \leq \frac{c}{\left\|w_{1}-w_{2}\right\|^{d-2}} \frac{1}{\left\|w_{3}-w_{4}\right\|^{d-2}}
$$

which proves the claim. By Lemma 5.6, the lace $\tilde{L}$ is acyclic. Therefore, the value of its diagram is bounded by $\tilde{C}^{(2)}\left(y_{1}, y_{2}, z_{1}, z_{2}\right)$, by Case 1 .

By Cases $1-3$, it follows that (7.8) holds when $\Delta=2$. We assume henceforth that $\Delta \geq 3$.

Case 4: Acyclic Laces Let $L$ be an acyclic lace. By Lemma 5.5, there is a branch $e$ such that there is a unique bond in $L$ that covers 0 and has an endpoint on branch $e$, with the restriction $\tilde{L}$ of $L$ to the other branches being a lace. Assume that $e=\Delta$. We show that it is possible to bound the value of the diagram corresponding to $L$ in terms of the diagram corresponding to $\tilde{L}$, leading to a recursive estimate.

The diagram variable corresponding to the unique bond with both endpoints on branch $\Delta$ is $y_{\Delta}$. Let $z_{\Delta}$ denote the diagram variable of the bond $b^{(\Delta)} \in L$ associated to branch $\Delta$. Let $w_{1}$ and $w_{2}$ be the variables adjacent to $z_{\Delta}$ on the branch which is different from $\Delta$. Then $w_{1}$ and $w_{2}$ are chosen from among 0 and $y_{j}, z_{j}$ with $1 \leq j<$ $\Delta$. Since either $\frac{1}{2}\left\|w_{1}-w_{2}\right\| \leq\|\| w_{2}-z_{\Delta} \| \mid$ or $\frac{1}{2}\left\|w_{1}-w_{2}\right\| \mid \leq\left\|w_{1}-z_{\Delta}\right\| \|$, it follows 
that

$$
\begin{aligned}
& \frac{1}{\left\|w_{1}-z_{\Delta}\right\| \|^{d-2}} \frac{1}{\left\|w_{2}-z_{\Delta}\right\| \|^{d-2}} \\
& \quad \leq \frac{c}{\left\|w_{1}-w_{2}\right\|^{d-2}}\left[\frac{1}{\left\|w_{1}-z_{\Delta}\right\|^{d-2}}+\frac{1}{\left\|w_{2}-z_{\Delta}\right\|^{d-2}}\right] .
\end{aligned}
$$

The factor $\left\|w_{1}-w_{2}\right\|^{2-d}$ contributes to the diagram corresponding to $\tilde{L}$, leaving additional factors

$$
\frac{1}{\left\|y_{\Delta}\right\| \|^{d-2}} \frac{1}{\left\|y_{\Delta}-z_{\Delta}\right\|^{2(d-2)}}\left[\frac{1}{\left\|w_{1}-z_{\Delta}\right\|^{d-2}}+\frac{1}{\left\|w_{2}-z_{\Delta}\right\|^{d-2}}\right]
$$

that contribute terms $Q$ appearing in the definition of $B^{(\Delta)}$ of (6.8). The restrictions on the domain of summation of the $z_{i}$, due to $L \in \tilde{\mathcal{L}}_{\mathcal{A}, \bar{x}}$, can be relaxed to summation over $\bar{z} \in B(\bar{x})$ in an upper bound, as in Case 2.

Case 5: Non-Minimal Cyclic Laces Let $L$ be a non-minimal cyclic lace. By Lemma 5.6, there is a bond $b$ whose removal leaves an acyclic lace $\tilde{L}$. It then follows, as in Case 3 , that the value of the diagram corresponding to $L$ is bounded above by a multiple of the value of the diagram corresponding to $\tilde{L}$.

Recursive Bound Given a diagram corresponding to an acyclic or non-minimal cyclic lace, we apply Cases $4-5$ repeatedly until we produce a minimal cyclic lace or we reduce the degree to 2 . Assume that the branches are ordered in such a way that whenever a branch is removed in applying Case 4 , it has the largest label. Then if $j$ is the branch removed, we have that each of the vertices $w_{i}(i=1,2)$ discussed in Case 4 is in $\left\{0, y_{1}, \ldots, y_{j-1}, z_{1}, \ldots, z_{j-1}\right\}$. This gives the desired result, where the sum over permutations allows for arbitrary orderings of the branches when applying Case 4.

\section{Proof of Proposition 2.7}

From the definitions in (2.22), it follows that

$$
\left|V_{\Delta}-\tilde{V}_{\Delta, \bar{x}}^{\mathrm{in}}\right| \leq \sum_{\bar{y} \in \mathbb{Z}^{d \Delta}}\left|\pi^{(\Delta)}(\bar{y})-\tilde{\pi}_{\bar{x}}^{(\Delta)}(\bar{y})\right|+\sum_{\bar{y} \notin B(\bar{x})}\left|\tilde{\pi}_{\bar{x}}^{(\Delta)}(\bar{y})\right| .
$$

Given $\bar{y} \notin B(\bar{x})$, there is a component $y_{e} \notin B\left(x_{e}\right)$. We perform an unrestricted sum over the other components of $\bar{y}$ and apply (2.19), to see that the second term on the right side of (8.1) is bounded by $c \sum_{e=1}^{\Delta}\left\|x_{e}\right\|^{4-d}$, as required.

To bound the first term in (8.1) we use (2.12) to write

$$
\pi^{(\Delta)}(\bar{y})-\tilde{\pi}_{\bar{x}}^{(\Delta)}(\bar{y})=\sum_{\bar{m} \in \mathbb{Z}_{+}^{\Delta}} \sum_{\omega \in \Omega_{\mathcal{A}}(\bar{y})} W_{z_{c}}(\omega)\left[J_{\mathcal{A}}(\omega)-\tilde{J}_{\mathcal{A}, \bar{x}}(\omega)\right] .
$$


To analyse (8.2), we rewrite $J_{\mathcal{A}}-\tilde{J}_{\mathcal{A}, \bar{x}}$ as follows. Since $\tilde{\mathcal{L}}_{\mathcal{A}, \bar{x}} \subset \mathcal{L}_{\mathcal{A}}$, it follows from (5.2) that

$$
J_{\mathcal{A}}=\sum_{L \in \tilde{\mathcal{L}}_{\mathcal{A}, \bar{x}}}\left[\prod_{b \in L} U_{b}\right] \prod_{b \in \mathcal{C}_{\mathcal{A}}(L)}\left[1+U_{b}\right]+\sum_{L \in \mathcal{L}_{\mathcal{A}} \backslash \tilde{\mathcal{L}}_{\mathcal{A}, \bar{x}}}\left[\prod_{b \in L} U_{b}\right] \prod_{b \in \mathcal{C}_{\mathcal{A}}(L)}\left[1+U_{b}\right]
$$

Since $\tilde{\mathcal{C}}_{\mathcal{A}, \bar{x}}(L) \subset \mathcal{C}_{\mathcal{A}}(L)$, in the first term of the right side of (8.3) we can rewrite

$$
\prod_{b \in \mathcal{C}_{\mathcal{A}}(L)}\left[1+U_{b}\right]=\prod_{b \in \tilde{\mathfrak{e}}_{\mathcal{A}, \bar{x}}(L)}\left[1+U_{b}\right]-\left[\prod_{b \in \tilde{\mathcal{C}}_{\mathcal{A}, \bar{x}}(L)}\left[1+U_{b}\right]\right]\left[1-\prod_{b \in \mathcal{C}_{\mathcal{A}}(L) \backslash \tilde{\mathcal{C}}_{\mathcal{A}, \bar{x}}(L)}\left[1+U_{b}\right]\right] .
$$

The contribution to the first term on the right side of (8.3) due to the first term of the right side of (8.4) is $\tilde{J}_{\mathcal{A}, \bar{x}}$. Therefore

$$
\begin{aligned}
J_{\mathcal{A}}-\tilde{J}_{\mathcal{A}, \bar{x}}= & \sum_{L \in \mathcal{L}_{\mathcal{A}} \backslash \tilde{\mathcal{L}}_{\mathcal{A}, \bar{x}}}\left[\prod_{b \in L} U_{b}\right] \prod_{b \in \mathcal{C}_{\mathcal{A}}(L)}\left[1+U_{b}\right] \\
& -\sum_{L \in \tilde{\mathcal{L}}_{\mathcal{A}, \bar{x}}}\left[\prod_{b \in L} U_{b}\right]\left[\prod_{b \in \tilde{\mathcal{C}}_{\mathcal{A}, \bar{x}}(L)}\left[1+U_{b}\right]\right]\left[1-\prod_{b \in \mathcal{C}_{\mathcal{A}}(L) \backslash \tilde{\mathcal{C}}_{\mathcal{A}, \bar{x}}(L)}\left[1+U_{b}\right]\right] .
\end{aligned}
$$

We insert (8.5) into (8.2), producing two terms. The first of these two terms can be written as $\sum_{N=1}^{\infty}(-1)^{N} \rho_{\bar{x}, N}^{(\Delta)}(\bar{y})$, where

$$
\rho_{\bar{x}, N}^{(\Delta)}(\bar{y})=\sum_{\bar{m} \in \mathbb{Z}_{+}^{\Delta}} \sum_{\omega \in \Omega_{\mathcal{A}}(\bar{y})} W_{z_{\mathrm{c}}}(\omega) \sum_{L \in \mathcal{L}_{\mathcal{A}}^{(N)} \backslash \tilde{\mathcal{L}}_{\mathcal{A}, \bar{x}}^{(N)}}\left[\prod_{b \in L}\left[-U_{b}\right]\right] \prod_{b \in \mathcal{C}_{\mathcal{A}}(L)}\left[1+U_{b}\right] .
$$

Similarly, for the second term of (8.5), we define

$$
\begin{aligned}
\mu_{\tilde{x}, N}^{(\Delta)}(\bar{y})= & \sum_{\bar{m} \in \mathbb{Z}_{+}^{\Delta}} \sum_{\omega \in \Omega_{\mathcal{A}}(\bar{y})} W_{z_{\mathcal{c}}}(\omega) \\
& \times \sum_{L \in \tilde{\mathcal{L}}_{\mathcal{A}, \bar{x}}^{(N)}}\left[\prod_{b \in L}\left[-U_{b}\right]\right]\left[\prod_{b \in \tilde{\mathcal{C}}_{\mathcal{A}, \bar{x}}(L)}\left[1+U_{b}\right]\right]\left[1-\prod_{b \in \mathcal{C}_{\mathcal{A}}(L) \backslash \tilde{\mathcal{C}}_{\mathcal{A}, \bar{x}}(L)}\left[1+U_{b}\right]\right] .
\end{aligned}
$$

Then (8.6) and (8.7) are both nonnegative, and

$$
\left|\pi^{(\Delta)}(\bar{y})-\tilde{\pi}_{\bar{x}}^{(\Delta)}(\bar{y})\right| \leq \sum_{N=1}^{\infty} \rho_{\bar{x}, N}^{(\Delta)}(\bar{y})+\sum_{N=1}^{\infty} \mu_{\bar{x}, N}^{(\Delta)}(\bar{y}) .
$$

We analyse $\rho_{\bar{x}, N}^{(\Delta)}$ using diagrams as in the bounds on $\tilde{\pi}$. Every $L \in \mathcal{L}_{\mathcal{A}} \backslash \tilde{\mathcal{L}}_{\mathcal{A}, \bar{x}}$ includes at least one bond $b$ in $\tilde{\mathcal{R}}_{\mathcal{A}, \bar{x}}=\mathcal{B}_{\mathcal{A}} \backslash\left[\tilde{\mathcal{V}}_{\mathcal{A}, \bar{x}} \cup \mathcal{H}_{\mathcal{A}}\right]$. This means that in the diagram corresponding to $L$, the variable $z$ associated to $b$ is summed over the set 
$B\left(x_{e}\right)^{c} \cup B\left(x_{f}\right)^{c}$, where $e$ and $f$ denote the branches containing the endpoints of $b$. We obtain a bound analogous to (6.9), except now the sum over $\bar{z}$ in (6.8) is replaced by a multiple of $\sum_{e=1}^{\Delta} \sum_{\bar{z} \in \mathbb{Z}^{d \Delta}: z_{e} \notin B\left(x_{e}\right)}$. Using a modification of (6.11) for $\rho$, we obtain the desired estimate

$$
\sum_{N=1}^{\infty} \sum_{\bar{y} \in \mathbb{Z}^{d \Delta}} \rho_{\bar{x}, N}^{(\Delta)}(\bar{y}) \leq c \sum_{e=1}^{\Delta} \sum_{z_{e} \notin B\left(x_{e}\right)} \frac{1}{\left\|z_{e}\right\|^{2 d-4}} \leq c \sum_{e=1}^{\Delta} \frac{1}{\left\|\mid x_{e}\right\|^{d-4}}
$$

It remains to estimate $\mu_{\bar{x}, N}^{(\Delta)}$. The difference between $\mu_{\bar{x}, N}^{(\Delta)}$ and $\tilde{\pi}_{\bar{x}, N}^{(\Delta)}$ resides in the indicator function $1-\prod_{b \in \mathcal{C}_{\mathcal{A}}(L) \backslash \tilde{\mathcal{C}}_{\mathcal{A}, \bar{x}}(L)}\left[1+U_{b}\right]$. The indicator function is nonzero only if there is a bond $b \in \mathcal{C}_{\mathcal{A}}(L) \backslash \tilde{\mathcal{C}}_{\mathcal{A}, \bar{x}}(L) \subset \tilde{\mathcal{R}}_{\mathcal{A}, \bar{x}}$ such that the endpoints of $b$ correspond to an intersection in the embedding. Thus $\mu_{\bar{x}, N}^{(\Delta)}$ can be bounded by diagrams in the same manner as $\tilde{\pi}_{\bar{x}, N}^{(\Delta)}$, but with an additional new intersection not present in the diagrams for $\tilde{\pi}_{\bar{x}, N}^{(\Delta)}$. In more detail, the modification to a diagram for $\tilde{\pi}_{\bar{x}, N}^{(\Delta)}$ is as follows. We select a pair of lines in a diagram bounding $\tilde{\pi}_{\bar{x}, N}^{(\Delta)}$, that lie between the branch point and the endpoint of the bond associated to the branch containing the line (since a bond $b \in \tilde{\mathcal{R}}_{\mathcal{A}, \bar{x}}$ must cover the branch point), and replace those lines by four lines that meet at a common vertex. This common vertex $w$ is summed over $B\left(x_{e}\right)^{c} \cup B\left(x_{f}\right)^{c}$, where $e$ and $f$ are the indices of the branches occurring in the bond $b$. It suffices to show that any new such diagram is bounded above by a small factor times a diagram that we have already bounded when estimating $\tilde{\pi}_{\bar{x}, N}^{(\Delta)}$. We can restrict our attention to basic diagrams, since by the above remarks the extra bond does not interfere with the reduction process of Section 7.2.

Let

$$
K(\bar{y}, \bar{z})=K_{0}(\bar{y}, \bar{z}) \frac{1}{\left\|w_{1}-w_{2}\right\|^{d-2}} \frac{1}{\left\|w_{3}-w_{4}\right\|^{d-2}}
$$

denote a term in $B^{(\Delta)}(\bar{y})$ with a specific pair of lines, from $w_{1}$ to $w_{2}$ and from $w_{3}$ to $w_{4}$, singled out. We assume that this pair of lines is a possible pair for the additional intersection described above. In bounding $\mu_{\bar{x}, N}^{(\Delta)}$, the term $K(\bar{y}, \bar{z})$ is replaced by

$$
K_{\mu}(\bar{y}, \bar{z})=K_{0}(\bar{y}, \bar{z}) \sum_{w \in B\left(x_{e}\right)^{c} \cup B\left(x_{f}\right)^{c}} \prod_{j=1}^{4} \frac{1}{\left\|w_{j}-w\right\|^{d-2}} .
$$

The sum on the right side of (8.11) can be replaced by $\sum_{w \in B\left(x_{e}\right)^{c}}+\sum_{w \in B\left(x_{f}\right)^{c}}$, in an upper bound. The two terms are similar and we consider only the first, in what follows. We divide the sums over $\bar{y}, \bar{z}$ into two cases, and show that each case satisfies the required bound.

Case 1 Assume that $w_{1}, w_{2}, w_{3}, w_{4}$ are inside $B\left(x_{e} / 2\right)$, so their norms are at most $\left|x_{e}\right| / 6$. Then we use $\left\|\left|w-w_{1}\right|\right\| \geq \frac{1}{6}\left\|x_{e}\right\|\left|\geq \frac{1}{2}\left\|w_{1}-w_{2}\right\|,\left\|\left|w-w_{3}\right|\right\| \geq\|w\|\right|-$

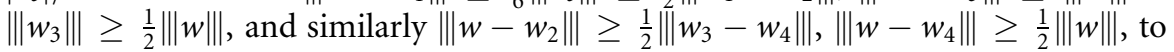


obtain

$$
\begin{aligned}
\sum_{w \in B\left(x_{e}\right)^{c}} \prod_{j=1}^{4} \frac{1}{\left\|w_{j}-w\right\|^{d-2}} & \leq \frac{c}{\left\|w_{1}-w_{2}\right\|^{d-2}} \frac{1}{\left\|w_{3}-w_{4}\right\|^{d-2}} \sum_{w \in B\left(x_{e}\right)^{c}} \frac{1}{\|w\|^{2 d-4}} \\
& \leq \frac{c}{\left\|w_{1}-w_{2}\right\|^{d-2}} \frac{1}{\left\|w_{3}-w_{4}\right\|^{d-2}} \frac{1}{\left\|x_{e}\right\|^{d-4}} .
\end{aligned}
$$

Arguing similarly when $w \in B\left(x_{f}\right)^{c}$, we obtain, as required,

$$
K_{\mu}(\bar{y}, \bar{z}) \leq c K(\bar{y}, \bar{z})\left(\frac{1}{\left\|x_{e} \mid\right\|^{d-4}}+\frac{1}{\left\|x_{f}\right\|^{d-4}}\right) .
$$

Case 2 Assume that one of $w_{1}, w_{2}, w_{3}, w_{4}$ is outside $B\left(x_{e} / 2\right)$. In this case we extend the sum over $w$ in (8.11) to a sum over all $w \in \mathbb{Z}^{d}$, and use (3.4) to bound it by $c\left|\left\|w_{1}-w_{2}\right\|\right|^{2-d}\left\|\mid w_{3}-w_{4}\right\|^{2-d}$. This gives the corresponding diagram for $\tilde{\pi}_{\bar{x}, N}^{(\Delta)}$, but with one vertex constrained to be large. This can then be bounded by summing (6.13) over $y$ or $z$ outside $B\left(x_{e} / 2\right)$, giving the desired result.

\section{Proof of Proposition 2.8}

Recall the definition of $\varphi_{\nu}(\vec{x})$ from (2.8). Recall from Section 2.2 that $\mathcal{G}_{\mathcal{N}}^{\text {err }}$ is the set of graphs $\Gamma \subset \mathcal{V}_{\mathcal{N}} \cup \mathcal{H}_{\mathcal{N}}$ such that $\mathcal{A}_{i}(\Gamma)=\mathcal{A}_{j}(\Gamma)$ for some $i \neq j, i, j \in S_{0}$. Let $E_{0}$ be the set of edges $e=\left(e_{1}, e_{2}\right)$ with $e_{1}, e_{2} \in S_{0}$. Note that $\mathcal{G}_{\mathcal{N}}^{\text {err }}$ is empty if $E_{0}$ is empty, so we assume that $E_{0} \neq \varnothing$. For $F \subset E_{0}$, we define $\mathcal{G}_{\mathcal{N}}^{\text {err }}(F)$ to be the set of graphs $\Gamma \in \mathcal{G}_{\mathcal{N}}^{\text {err }}$ such that for each $f=\left(f_{1}, f_{2}\right) \in F, \mathcal{A}_{f_{1}}(\Gamma)=\mathcal{A}_{f_{2}}(\Gamma)$. Note that branches in $E_{0} \backslash F$ may or may not have this property. By the inclusion-exclusion relation,

$$
\left|\sum_{\Gamma \in \mathcal{G}_{\mathcal{N}}^{\text {err }}} \prod_{b \in \Gamma} U_{b}\right| \leq \sum_{F \subset E_{0}: F \neq \varnothing}\left|\sum_{\Gamma \in \mathcal{G}_{\mathcal{N}}^{\text {err }}(F)} \prod_{b \in \Gamma} U_{b}\right| .
$$

It suffices to show that the contribution to (2.8) due to each term $\sum_{\Gamma \in \mathcal{G}_{\mathcal{N}}^{\text {err }}(F)} \prod_{b \in \Gamma} U_{b}$ obeys the bound of Proposition 2.8.

For $\Gamma \in \mathcal{G}_{\mathcal{N}}^{\text {err }}(F), e=\left(e_{1}, e_{2}\right) \in F$ and $i=1,2$, we define $\Gamma_{e, i}=\Gamma_{e, i}(\Gamma)$ to be the set of bonds $s t \in \Gamma$ that have an endpoint on branch $T_{e}$ and that cover $e_{i}$. By definition of $\mathcal{G}_{\mathcal{N}}^{\text {err }}(F), \Gamma_{e, i} \neq \varnothing$. We select a unique bond $j_{i} k_{i} \in \Gamma_{e, i}$ according to the procedure of Definition 5.1, with $j_{i} \notin T_{e}, k_{i} \in T_{e}$. We refer to $j_{i} k_{i}$ as the bond associated to $T_{e}$ near $e_{i}$. Given $\Gamma \in \mathcal{G}_{\mathcal{N}}^{\text {err }}(F)$ and $e \in F$, we construct a graph $P_{\Gamma}(e)=\left\{s_{1} t_{1}, \ldots, s_{l} t_{l}\right\} \subset \Gamma$ on a subnetwork containing $T_{e}$ as follows. We set $s_{1} t_{1}=j_{1} k_{1}$, and then perform the $T_{e}$-lace construction of Section 5.1 along $T_{e}$ in the direction from $e_{1}$ to $e_{2}$ until $k_{2}$ is covered, obtaining $s_{1} t_{1}, \ldots, s_{l-1} t_{l-1}$. Then we set $s_{l} t_{l}=k_{2} j_{2}$. Let

$$
\mathrm{P}_{\Gamma}=\bigcup_{e \in F} \mathrm{P}_{\Gamma}(e)
$$


Each $\mathrm{P}_{\Gamma}(e)$ induces a subnetwork $\mathcal{J}_{e}(\Gamma)$, which is either a path or a bubble, consisting of the path from $s_{1}$ to $t_{l}$ via $T_{e}$, i.e., the path $s_{1} \rightarrow e_{1} \stackrel{e}{\rightarrow} e_{2} \rightarrow t_{l}$ that contains no branch points except $e_{1}$ and $e_{2}$. This path may close up on itself, as depicted in Figure 11 .
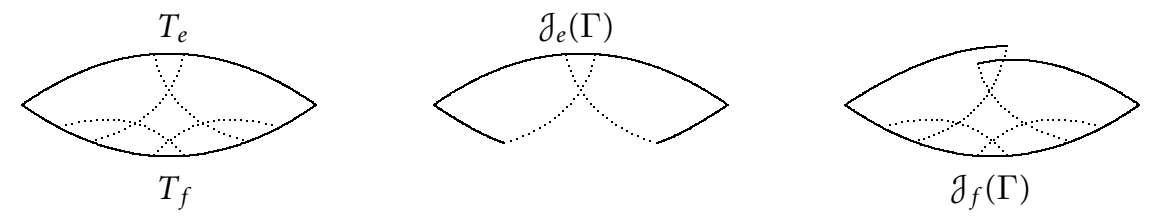

Figure 11: An example of $\Gamma \in \mathcal{G}_{\mathcal{N}}^{\text {err }}(F)$, with its corresponding $\mathcal{\partial}_{e}(\Gamma)$ and $\mathcal{J}_{f}(\Gamma)$, for $F=\{e, f\}$. A graph bond covers the interval on its concave side.

Now we resum $\sum_{\Gamma \in \mathcal{G}_{\mathcal{1}}^{\text {err }}(F)} \prod_{b \in \Gamma} U_{b}$ as in (5.2). Let $e \in F$, and let $a_{i}$ be a path point on one of the branches adjacent to $T_{e}$ at $e_{i}(i=1,2)$. Let $\mathcal{J}_{e}\left(a_{1}, a_{2}\right)$ denote the path or bubble determined by travelling from $a_{1}$ to $a_{2}$ via $T_{e}$, as above. We write $\mathcal{P}_{F, \vec{x}}=$ $\mathcal{P}_{F, \vec{x}}(\omega)$ for the set of all graphs $L=\bigcup_{e \in F} L_{e}$ for which the collection $\left(L_{e}: e \in F\right)$ satisfies the following two properties:

1. $L_{e}$ is a lace on $\mathcal{J}_{e}\left(a_{1}, a_{2}\right)$ for some $a_{1}, a_{2}$, where we regard $\mathcal{J}_{e}\left(a_{1}, a_{2}\right)$ as a "path" even in the case where it is a bubble. See Figure 11. In addition, the only bond in $L_{e}$ that covers $e_{i}$ is the bond in $L_{e}$ that is associated to $T_{e}$ near $e_{i}(i=1,2)$.

2. If $b \in L_{e}$ is associated to $T_{e}$ near $e_{i}$ among bonds in $L_{e}$, then, among all bonds in $L$, it is associated to $T_{e}$ near $e_{i}$.

The decomposition of $L \in \mathcal{P}_{F, \vec{x}}$ into constituents $L_{e}$ is then unique. Given $L \in \mathcal{P}_{F, \vec{x}}$, we define $Q_{F, \vec{x}}(L)$ to be the set of bonds $b \in \mathcal{V}_{\mathcal{N}} \cup \mathcal{H}_{\mathcal{N}}$ that are compatible with $L$ in the sense that $P_{L \cup\{b\}}=L$. We will not need a precise description of the set $Q_{F, \vec{x}}(L)$, only some of its simple properties. First, we need that similarly to the lace $\mathrm{L}_{\Gamma}$ defined in Section 5.1, given $\Gamma \in \mathcal{G}_{\mathcal{N}}^{\text {err }}(F)$, we have $\mathrm{P}_{\Gamma}=L$ if and only if $L \subset \Gamma$ is a graph in $\mathcal{P}_{F, \vec{x}}$ and $\Gamma \backslash L \subset Q_{F, \vec{x}}(L)$. This follows as in [5, Proposition 2.6], because the bonds in $\mathrm{P}_{\Gamma}$ are selected from $\Gamma$ according to some optimality conditions, and $Q_{F, \vec{x}}(L)$ is precisely the set of bonds whose presence does not destroy these optimality conditions. Therefore, by resumming as in (5.2), we obtain

$$
\sum_{\Gamma \in \mathcal{S}_{\mathcal{N}}^{\operatorname{err}}(F)} \prod_{b \in \Gamma} U_{b}=\sum_{L \in \mathcal{P}_{F, \bar{x}}}\left[\prod_{b \in L} U_{b}\right] \prod_{b \in \mathbb{Q}_{F, \bar{x}}(L)}\left[1+U_{b}\right] .
$$

Now that the resummation has been performed and the interaction has been partially restored via the compatible bonds, we may take absolute values inside sums.

The contribution to $\varphi_{\nu}(\vec{x})$ due to the term $\left|\sum_{\Gamma \in \mathcal{G}_{\mathcal{N}}^{\text {err }}(F)} \prod_{b \in \Gamma} U_{b}\right|$ in (9.1) is thus bounded by

$$
\sum_{N=2}^{\infty} \sum_{\vec{n} \in \mathbb{N}^{|E|}} \sum_{\omega \in \Omega_{\mathcal{N}}(\vec{x})} W_{z_{\mathcal{c}}}(\omega) \sum_{L \in \mathcal{P}_{F, \bar{x}}^{(N)}}\left[\prod_{b \in L}\left[-U_{b}(\omega)\right]\right] \prod_{b \in Q_{F, \bar{x}}(L)}\left[1+U_{b}(\omega)\right],
$$


where $\mathcal{P}_{F, \vec{x}}^{(N)}$ is the set of graphs in $\mathcal{P}_{F, \vec{x}}$ consisting of exactly $N$ bonds. To proceed we need a second property of $Q_{F, \vec{x}}(L)$, which we now describe. The branch points of the network together with the endpoints of bonds in $L$ subdivide $\mathcal{N}$ into intervals, as in the discussion following (7.2). Any bond that has both endpoints in one of these intervals lies in $Q_{F, \vec{x}}$, again because of the way $\mathrm{P}_{\Gamma}$ was defined. For example, for each $e \in E$ such that $T_{e}^{\circ}$ is disjoint from $\mathcal{\partial}_{f}(L)$ for all $f \in F$, the path $T_{e}$ comprises an interval as mentioned above, and we have $\mathcal{H}_{\mathcal{N}, e} \subset Q_{F, \vec{x}}(L)$. All other $T_{e}$ are broken up into smaller intervals. It follows that in (9.4) we can restrict the sum over $\omega$ so that each interval is embedded as a self-avoiding walk. We bound the contribution of all other $b \in Q_{F, \vec{x}}$ using $1+U_{b} \leq 1$.

As in Section 7.1, (9.4) is bounded by $\sum_{\mathcal{F} \in \mathcal{D}_{F}^{(N)}(\vec{x})} A(\mathcal{F})$, where $\mathcal{D}_{F}^{(N)}(\vec{x})$ is a set of diagrams. An example of a diagram is given in Figure 12. Diagrams can be quite complicated when $\nu$ is large and $F$ is large. Fortunately, it will not be necessary in the proof to understand the detailed structure of these diagrams. Instead, we will apply a reduction process that allows us to analyse in detail just two special cases.
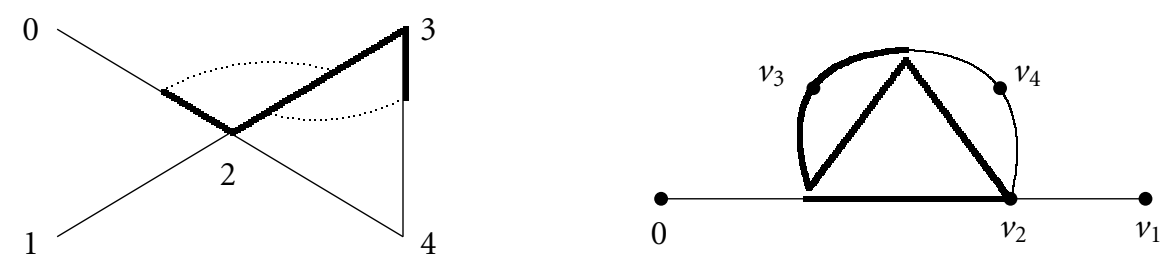

Figure 12: An example of an $L \in \mathcal{P}_{F, \vec{x}}^{(2)}$ with $F=(2,3)$, together with the diagram in $\mathcal{D}_{F}^{(2)}(\vec{x})$ that bounds its contribution to $\varphi_{\nu}(\vec{x})$.

A power of $\beta$ will control the sum over $N$ of the diagrams, as before. To see this, given $L \in \mathcal{P}_{F, \vec{x}}$, let $N^{(e)}$ denote the number of bonds in $L_{e}$ that have both endpoints on $T_{e}$. Using the fact that $L_{e}$ is a lace, it is not hard to show that at least $N^{(e)}-1$ of the subintervals of $T_{e}$ induced by $L_{e}$ have positive length (for a lower bound, we count only the subintervals on $T_{e}$ that are not covered by the bonds in $L_{e}$ that cover $e_{1}$ and $e_{2}$ ). Thus the exponent of $\beta$ is at least

$$
\sum_{e \in F} \max \left\{0, N^{(e)}-1\right\} \geq \max \{0, N-\text { const. }\},
$$

where the constant only depends on $\nu$. We will estimate the diagrams by reducing them to one of two simple diagrams. The number of diagrams in $\mathcal{D}_{F}^{(N)}(\vec{x})$ can be shown to be bounded by $c^{N}$, so this is still controlled by the power of $\beta$.

Fix an arbitrary $f \in F$. Given $L \in \mathcal{P}_{F, \vec{x}}$, let $L_{f}=\mathrm{P}_{L}(f) \subset L$ be the corresponding lace on $\mathcal{\partial}_{f}(L)$. We obtain an upper bound by eliminating all bonds st $\in L \backslash L_{f}$. Indeed, it follows from an application of (3.4) that the elimination of a bond from a lace gives rise to a diagram that is an upper bound on the diagram corresponding to the original lace, up to a constant multiple (compare the application of (3.4) in 
Case 3 of Section 7.3). This is natural, since a diagram with an additional intersection should not be larger than a diagram in which the intersection is not required. We may therefore restrict attention to the case $L=L_{f}$. There are two possibilities, depending on whether $\partial_{f}(L)$ is a path or a bubble, as in Figure 11.

Suppose that $L=L_{f}$, and consider first the case where $\mathcal{J}_{f}(L)$ is a path. In this case, $L=\left\{s_{1} t_{1}, \ldots, s_{l} t_{l}\right\}$, with $s_{1} t_{1}$ and $s_{l} t_{l}$ associated to $T_{f}$ near the endpoints $f_{1}, f_{2}$ of $f$. We claim that, up to a constant $c^{l}$ (which is compensated by a power of $\beta$ ), the diagram corresponding to $L$ is bounded above by the diagram corresponding to the lace with just two bonds. To see this, suppose $l \geq 3$, let $w_{i}=\omega\left(s_{i}\right)=\omega\left(t_{i}\right)$ for $i=1,2,3, w=\omega\left(f_{1}\right)$, and let $u$ be the variable adjacent to $t_{2}$ in the direction of $t_{l}$ (so $u=w_{4}$ if $l \geq 4$ and $u=\omega\left(f_{2}\right)$ if $\left.l=3\right)$. See Figure 13. By (3.4),

$$
\begin{aligned}
& \sum_{w_{2} \in \mathbb{Z}^{d}} \frac{1}{\left\|w_{2}-w\right\|^{d-2}} \frac{1}{\left.\left\|w_{2}-w_{1}\right\|\right|^{d-2}} \frac{1}{\left\|w_{2}-w_{3}\right\|^{d-2}} \frac{1}{\left\|w_{2}-u\right\|^{d-2}} \\
& \quad \leq \frac{c}{\left\|w-w_{3} \mid\right\|^{d-2}} \frac{1}{\left\|w_{1}-u\right\|^{d-2}},
\end{aligned}
$$

and this estimate can be applied recursively to prove the claim. It remains to bound the diagram corresponding to the lace with two bonds. In this diagram there is a factor $\left\|x_{e}\right\| \|^{2-d}$ for each $e \in E$ such that $\mathcal{J}_{f}(L) \cap T_{e}^{\circ}=\varnothing$. There are either one or two edges $e \neq f$, such that $\mathcal{J}_{f}(L) \cap T_{e}^{\circ} \neq \varnothing$. For any such edge the portion disjoint from $\mathcal{J}_{f}(L)$ contributes a factor bounded by $c\left\|\mid x_{e}\right\|^{2-d}$, since both bonds in $L$ are in $\mathcal{V}_{\mathcal{N}}$.

Thus the diagram corresponding to the lace with two bonds makes a contribution

$$
\begin{aligned}
& {\left[\prod_{e \in E \backslash\{f\}} \frac{1}{\left\|x_{e}\right\|^{d-2}}\right]} \\
& \quad \times \sum_{z_{1}, z_{2} \in B\left(x_{f}\right)} \frac{1}{\left\|z_{1}\right\|^{d-2}} \frac{1}{\left\|z_{2}\right\|^{d-2}} \frac{1}{\left\|x_{f}-z_{1}\right\|^{d-2}} \frac{1}{\left\|x_{f}+z_{2}\right\|^{d-2}} \frac{1}{\left\|x_{f}+z_{2}-z_{1}\right\|^{d-2}},
\end{aligned}
$$

and the summation is bounded, as required, by a multiple of $\left\|x_{f}|\||^{4-3(d-2)}=\right.$ $\left\|\left.\left|x_{f}\right|\right|^{-(d-2)}\right\|\left|x_{f} \|\right|^{-2(d-4)}$.

A similar analysis can be applied in the case where $\partial_{f}(L)$ is a bubble, rather than a path. Let $f^{\prime}$ denote the edge which, together with $f$, comprises the bubble. This time the reduced lace gives rise to the diagram with value

$$
\begin{aligned}
& {\left[\prod_{e \in E \backslash\left\{f, f^{\prime}\right\}} \frac{1}{\left\|x_{e}\right\|^{d-2}}\right]} \\
& \quad \times \sum_{z_{1}, z_{2} \in B\left(x_{f}\right)} \frac{1}{\left.\left\|x_{f}-z_{1}\right\|\right|^{2(d-2)}} \frac{1}{\left\|x_{f}+z_{2}\right\|^{2(d-2)}} \frac{1}{\left\|x_{f}+z_{2}-z_{1}\right\|^{2(d-2)}},
\end{aligned}
$$

and the summation is bounded by a multiple of

$$
\left\|\left|x_{f}\left\|^{2 d-6(d-2)}=\right\|\right| x_{f}\right\|^{-2(d-2)}\left\|\left|x_{f} \|\right|^{-2(d-4)},\right.
$$


(a)
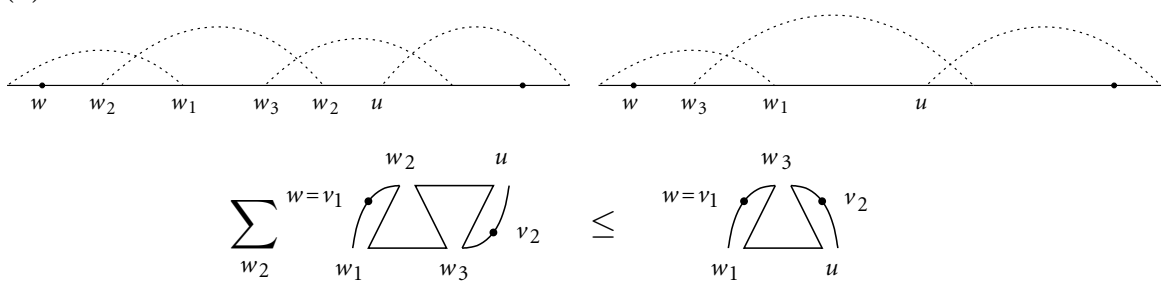

(b)
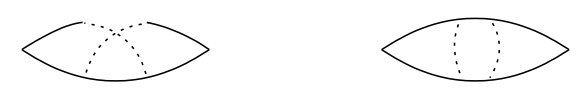

Figure 13: (a) Diagrammatic representation of the reduction step (9.6). Vertex labels on the upper figure represent the lattice sites in $\mathbb{Z}^{d}$ to which the vertices are embedded. (b) The two-bond laces corresponding to the sums over $z_{1}, z_{2}$ in (9.7) and (9.8), respectively.

as required.

Acknowledgements This work was supported in part by NSERC of Canada. The work of AAJ was also supported by a Postdoctoral Fellowship from the Pacific Institute for the Mathematical Sciences. We thank Remco van der Hofstad and an anonymous referee for helpful suggestions.

\section{References}

[1] D. C. Brydges and T. Spencer, Self-avoiding walk in 5 or more dimensions. Commun. Math. Phys. 97(1985), 125-148.

[2] B. Duplantier, Statistical mechanics of polymer networks of any topology. J. Statist. Phys. 54(1989), 581-680.

[3] T. Hara, Critical two-point functions for nearest-neighbour high-dimensional self-avoiding walk and percolation. In preparation.

[4] T. Hara, R. van der Hofstad and G. Slade, Critical two-point functions and the lace expansion for spread-out high-dimensional percolation and related models. Ann. Probab. 31(2003), 349-408.

[5] R. van der Hofstad and G. Slade, The lace expansion on a tree with application to networks of self-avoiding walks. Adv. Appl. Math. 30(2003), 471-528.

Department of Mathematics University of British Columbia Vancouver, BC

V6T $1 Z 2$

email: holmes@math.ubc.ca tetsu@math.ubc.ca slade@math.ubc.ca
Centrum voor Wiskunde en Informatica

P. O. Box 94079

NL-1090 GB Amsterdam

The Netherlands

email: Antal.Jarai@cwi.nl 